\title{
Point mutations in murine Nkx2-5 phenocopy human congenital heart disease and induce pathogenic Wnt signaling
}

Milena B. Furtado, ${ }^{1,2}$ Julia C. Wilmanns, ${ }^{2,3}$ Anjana Chandran, ${ }^{2}$ Joelle Perera, ${ }^{2}$ Olivia Hon, ${ }^{1}$ Christine Biben, ${ }^{4}$ Taylor J. Willow, ${ }^{1}$ Hieu T. Nim, ${ }^{2}$ Gurpreet Kaur, ${ }^{2}$ Stephanie Simonds, ${ }^{5}$ Qizhu Wu, ${ }^{6}$ David Willians, ${ }^{7}$ Ekaterina Salimova, ${ }^{2}$ Nicolas Plachta, ${ }^{8}$ James M. Denegre, ${ }^{1}$ Stephen A. Murray, ${ }^{1}$ Diane Fatkin,, ${ }^{9,10,11}$ Michael Cowley, ${ }^{5}$ James T. Pearson,, ${ }^{5,6}$ David Kaye, ${ }^{7}$ Mirana Ramialison, ${ }^{2}$ Richard P. Harvey, ${ }^{10,12}$ Nadia A. Rosenthal, ${ }^{1,2,13}$ and Mauro W. Costa ${ }^{1,2}$

${ }^{1}$ The Jackson Laboratory, Bar Harbor, Maine, USA. ${ }^{2}$ Australian Regenerative Medicine Institute, Monash University, Clayton, Australia. ${ }^{3}$ Department of Cardiology and Angiology, Medical School Hannover, Hannover, Germany. ${ }^{4}$ The Walter and Eliza Hall Institute of Medical Research, Parkville, Australia. ${ }^{5}$ Department of Physiology, ${ }^{6}$ Monash Biomedical Imaging, Monash University, Clayton, Australia. ${ }^{7}$ Heart Failure Research Group, Baker IDI Heart and Diabetes Institute, Melbourne, Australia. ${ }^{8}$ Institute of Molecular and Cell Biology, A*STAR, Singapore. ${ }^{9}$ Molecular Cardiology, Victor Chang Cardiac Research Institute, Darlinghurst, Australia. ${ }^{10} \mathrm{Faculty}$ of Medicine and School of Biological and Biomolecular Sciences, University of New South Wales, Kensington, Australia. "Cardiology Department, St. Vincent's Hospital, Darlinghurst, Australia. ${ }^{12}$ Stem Cell Biology Division, Victor Chang Cardiac Research Institute, Darlinghurst, Australia. ${ }^{13}$ National Heart and Lung Institute, Imperial College, London, United Kingdom.

Mutations in the Nkx2-5 gene are a main cause of congenital heart disease. Several studies have addressed the phenotypic consequences of disrupting the Nkx2-5 gene locus, although animal models to date failed to recapitulate the full spectrum of the human disease. Here, we describe a new Nkx2-5 point mutation murine model, akin to its human counterpart disease-generating mutation. Our model fully reproduces the morphological and physiological clinical presentations of the disease and reveals an understudied aspect of Nkx2-5-driven pathology, a primary right ventricular dysfunction. We further describe the molecular consequences of disrupting the transcriptional network regulated by Nkx2-5 in the heart and show that Nkx2-5-dependent perturbation of the Wnt signaling pathway promotes heart dysfunction through alteration of cardiomyocyte metabolism. Our data provide mechanistic insights on how Nkx2-5 regulates heart function and metabolism, a link in the study of congenital heart disease, and confirms that our models are the first murine genetic models to our knowledge to present all spectra of clinically relevant adult congenital heart disease phenotypes generated by NKX2-5 mutations in patients.

Authorship note: MBF and JCW contributed equally to this work. NAR and MWC contributed equally to this work.

Conflict of interest: The authors have declared that no conflict of interest exists.

Submitted: May 6, 2016

Accepted: February 1, 2017

Published: March 23, 2017

Reference information:

JCI Insight. 2017;2(6):e88271. https:// doi.org/10.1172/jii.insight.88271

\section{Introduction}

Congenital Heart Disease is the most prevalent of all clinically relevant human birth defects, affecting approximately $1 \%$ of children (1). Much higher rates are actually scored if milder clinically silent defects in childhood are accounted for, including bicuspid aortic valve, aneurism of the atrial septum, and persistent left superior vena cava (2). Advances in prenatal diagnosis, improved surgical procedures, and aftercare in modern medicine have led to a continual increase in survival rates of patients bearing congenital cardiac malformations. More recent data places the number of adults presenting some form of congenital heart disease worldwide at approximately 21 million (3). Congenital heart disease survivors have significant improvements in quality of life, reach adulthood, and start new families, and as a consequence, modern society is experiencing an annual increase in the proportion of genetically predisposed individuals who can develop cumulative and severe cardiac dysfunctions (4).

The homeobox transcription factor $N k \times 2-5$ is recognized as one of the pivotal genes associated with heart defects (5). Nkx2-5 expression is highly evolutionarily conserved and is essential for heart development 
and determination of myocardial cell fate (6). Absence of Nkx2-5 in mice results in impaired cardiac growth and chamber formation, deranged gene regulatory networks, and early embryonic lethality (7, 8). Deletion at postnatal stages has shown that $N k \times 2-5$ activity is essential for maintenance of the cardiac conduction system (9-12). Overexpression of Nkx2-5 also underpins conduction defects in myotonic dystrophy in a mouse model (13).

Mutations in the NKX2-5 human gene have an incidence of $\sim 4 \%$ of all congenital heart diseases in patients (5), being one of the most common genetic causes of congenital heart disease, and are associated with severe structural and functional cardiac impairment. More than 50 NKX2-5 mutations have been associated with a diverse range of heart muscle, septal, and conduction system defects (14-20). Patients with a wide range of heterozygous $N K X 2-5$ mutations display diverse cardiac abnormalities that include septal defects, conotruncal malformations, hypoplastic left heart, dilated cardiomyopathy, and atrioventricular conduction block (5). Long-term followup of patients carrying NKX2-5 mutations show 94\% prevalence of atrioventricular block with increased atrial fibrillation episodes and high incidence of sudden death, highlighting the importance of this gene in adult life and long-term followup, especially given the aging cohort of patients carrying congenital heart disease (21). We have recently uncovered a NKX2-5 mutation in a family that showed septal malformations, left ventricular noncompaction, dilated cardiomyopathy, and conduction defects over 3 generations (22). This mutation creates an isoleucine to methionine (I-M) change at position 184 of the protein homeodomain region, critical for NKX2-5 function. This mutation is equivalent to position 183 in mice. Mutations in this residue were also recently found in two new independent families that share most landmarks seen in our original findings. These landmarks included the presence of dilated cardiomyopathy, normally not seen in other families harboring NKX2-5 mutations (23). Biochemical characterization of this protein showed that mutations in this residue lead to decreased binding and transcriptional activity (22).

Most current data regarding NKX2-5 dysfunction in disease were obtained using mouse models containing partial/full deletion or overexpression of mutated Nkx2-5 (9-13, 24). These models have been proven too severe or only displayed partial penetrance of the spectrum of phenotypic changes observed in patients, providing only marginal insights into the mechanisms of action of Nkx2-5 in disease generation. Specifically, such models did not reproduce the human condition because it is likely caused by partial disruption of protein function $(22,25)$.

Here, we describe a $N k \times 2-5$ knockin mouse model that faithfully reproduces most of the characteristics of NKX2-5 mutations in patients and uncovers an important and unexplored link between Nkx2-5 regulation of the Wnt signaling pathway and late-onset adult congenital heart disease.

\section{Results}

NKX2-5 I-M and I-P mutant proteins display decreased affinity for native DNA binding sites. We have previously reported a novel familial I-M mutation at the highly conserved position 184 of the homeodomain region of the NKX2-5 protein. Affected members of this family displayed most of the classical clinical characteristics described for NKX2-5 gene disruption (22). The mutation showed complete correlation with development of the phenotype, including a previously undetected ventricular dilation. Biochemical characterization of the I-M mutant protein showed decreased DNA binding affinity and partial transcriptional impairment. In contrast, mutation of the same residue to a proline (I-P) led to a much more severe functional impairment of protein activity in vitro (22). This latter change has been previously used to test the effect of the overexpression of a "dominant negative" form of Nkx2-5 in mice, causing ventricular dilation and mortality associated with severe arrhythmic episodes (26).

Although important when defining the biochemical properties of mutant proteins, in vitro assays (22, $25,27)$ are somewhat limited in spectrum, as changes in binding affinity and/or transcriptional activity are only tested in selected targets and cannot address the overall changes observed in native DNA sites. To overcome this issue, we analyzed the kinetics of murine I-M and I-P NKX2-5 mutant proteins using multiphoton-based photoactivation and fluorescence correlation spectroscopy (28) (Figure 1).

While photoactive GFP (paGFP) shows free diffusion, interpreted as no DNA binding (Figure 1B and Supplemental Table 1; supplemental material available online with this article; https://doi.org/10.1172/ jci.insight.88271DS1), NKX2-5 WT protein shows anomalous slow diffusion parameter, indicative of protein-DNA binding in the HL-1 nucleus. Dwell time of the slow diffusion component for NKX2-5 WT was $108.1 \pm 9.98$. NKX2-5 I183M-paGFP protein displayed reduced dwell time $(65.21 \pm 6.71)$, reflecting 
A

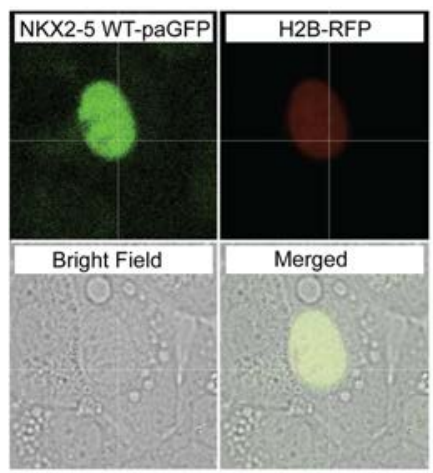

B

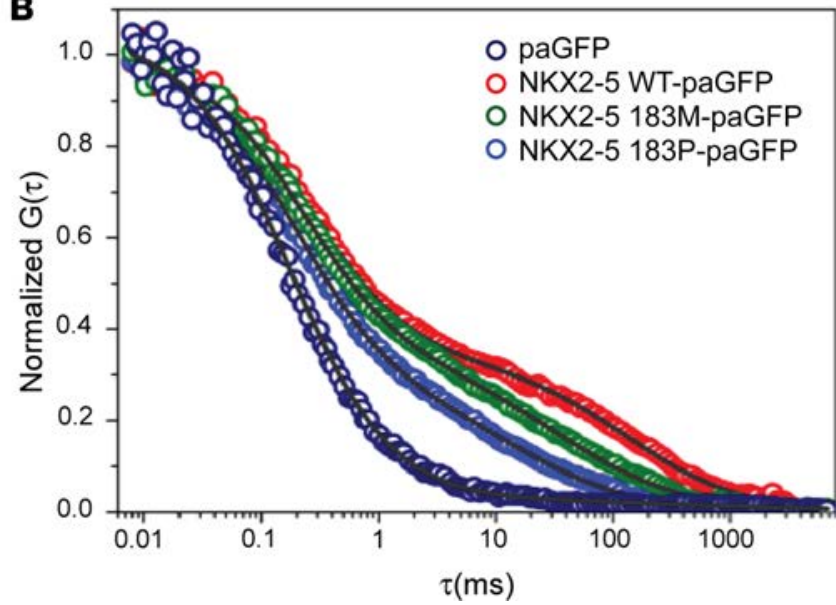

Figure 1. NKX2-5 mutant protein displays decreased affinity for its endogenous DNA binding sites. In situ analysis of DNA binding in transiently transfected $\mathrm{HL}-1$ cardiomyocytes confirms decreased affinity of Nkx2-5 I184M and P for target sites. (A) Example of nucleus analyzed by photoactivation; H2B-RFP fluorescence (red) colocalizes with Nk2-5-paGFP-transcfected (green) cells, while GFP can only be visualized after photoactivation. (B) Typical autocorrelation curves for paGFP and NKX2-5 proteins (WT, 183M, and 183P), showing fast and slow diffusion components for each protein. Note anomalous diffusion for NKX2-5 mutant proteins. pa, photoactivatable; RFP, red fluorescent protein; $\mathrm{G}$, fluctuations in fluorescence; $\tau$, time in milliseconds.

decreased DNA binding to endogenous genome targets. NKX2-5 I183P-paGFP had even lower dwell time (34.03 \pm 3.06$)$, consistent with a more severe impairment of the DNA binding domain by the I-P change and predicted structural changes in the homeodomain region (22). These experiments confirm that the mutant proteins display a significant reduction in DNA binding affinity to endogenous sites in situ and therefore must lead to a wide array of changes in transcription regulatory networks in vivo, corroborating our biochemical observations for patients with familial congenital heart disease (22).

Homozygous Nkx2-5 point mutations are embryonic lethal and phenocopy-null models. As previously mentioned, current disease murine models for $N k \times 2-5$ do not fully reproduce the human condition; therefore, proper models to study the mechanisms associated with the development of cardiac dysfunction are needed. To analyze the transcriptional activity and consequent phenotype of NKX2-5 mutant proteins in vivo, we engineered point mutations at the I183 residue (equivalent to I184 in humans), replacing it with either the original isoleucine sequence (Nkx2-5 control), I to M (c-g) or I to P (atc$\mathrm{cca}$ ) that lead to methionine or proline residue changes at 183 position (Figure 2A and Supplemental Figure 1). Genomic targeting was confirmed by Southern blot and sequencing of recombineered mice (Figure 2B and Supplemental Figure 1).

To determine the relative strength of the $\mathrm{M}$ and $\mathrm{P}$ mutations, $N k \times 2-5^{183 M /+}$ or $N k \times 2-5^{183 P /+}$ heterozygous mice were intercrossed for Mendelian birth ratio analysis. $N k \times 2-5$ null mice $\left(N k \times 2-5^{4 / 4}\right)$ die around 10.5 days after coitum in embryonic development $(7,8,29)$. No homozygous $N k x 2-5^{183 M / 183 M}$ or $N k \times 2-5^{183 P / 183 P}$ offspring were obtained, while mice in the control line $N k \times 2-5^{C / C}$ were born with expected ratios (Supplemental Table 2). In developmental analyses, no mutant $N k \times 2-5^{183 M / 183 M}$ or $N k \times 2-5^{183 P / 183 P}$ embryos were found past 11.5 days after coitum. At 10.5 days after coitum, embryos displayed severe growth retardation and heart abnormalities (Figure 3A), whereas embryos were viable at 9.5 days after coitum but displayed dysmorphic hearts arrested at the looping stage. This phenotype was more pronounced in $N k \times 2-5^{183 P / 183 P}$ when compared with $N k \times 2-5^{183 M / 183 M}$ embryos. No defects were observed in heterozygous embryos.

Expression levels of Nppa (a direct target of NKX2-5) were downregulated in the outer curvature of the heart in $N k \times 2-5^{183 M / 183 M}$ embryos at 9.5 days after coitum, while a residual expression in the atrial-forming regions was seen (Figure $3 \mathrm{~B}$ ). Consistent with the more severe phenotype observed in $N k \times 2-5^{183 P / 183 P}$ embryos, no significant expression of $N p p a$ was detected (Figure 3B). Mlc2v (a marker of the developing myocardium) was still present in both mutants, indicating that the initial steps of cardiac specification were intact, although subsequent cardiac morphogenesis, maturation, and function were arrested. Heterozygous $N k \times 2-5^{183 M /+}$ and $N k \times 2-5^{183 P /+}$ embryos showed normal Nppa and Mlc2v expression when compared with the WT littermates (Figure 3C). Our data highlight the severity of these homeodomain point mutations, which 


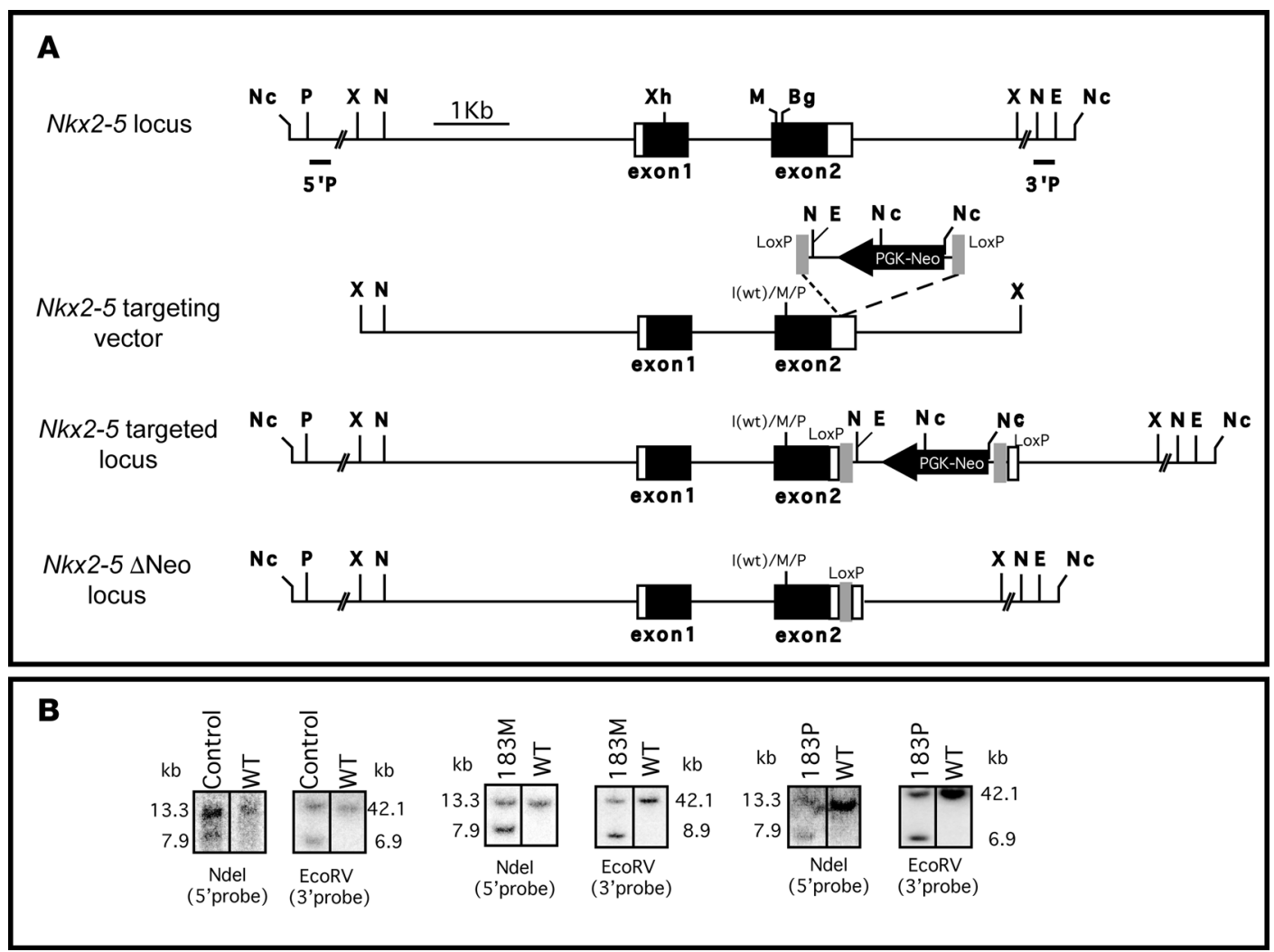

Figure 2. Targeting constructs and strategy for generation of $\mathbf{N k x 2 - 5}$ mouse knock-in model. (A) Diagram showing Nkx2-5 conditional mouse construct. (B) Southern blot analysis of targeted embryonic stem cells showing recombination using 5' and 3' probes. Nc, Ncol; P, Pvull; X, Xbal; N, Notl; Xh, Xhol; M, Mlul; Bg, Bglll; E, EchoRI; 5'P, 5'Southern probe; 3'P, 3' Southern probe; LoxP, Cre-recombinase cleavage sites; PGK-Neo, phosphoglycerate kinase promoter, neomycin resistance gene.

are as disruptive for normal heart development as the total lack of NKX2-5 protein and explain why only heterozygous mutations in the NKX2-5 gene are seen in patients (30).

Nkx2-5 heterozygous mutants develop adult-onset cardiac dysfunction. Although heterozygous mice carrying $N k \times 2-5 \mathrm{M}$ or P mutations were viable and fertile, a small decrease in the prevalence of mutant $N k \times 2-5^{183 M /+}$ or $N k \times 2-5^{183 P /+}$ when compared with $N k \times 2-5^{C /+}$ mice was observed, although not statistically significant (Supplemental Table 2). No differences in mortality rates after birth were found. Nevertheless, morphological analysis of adult hearts showed the presence of highly penetrant heart pathology in both mutant lines (Figure 4A and Supplemental Figure 2), presented as right ventricle and right atrial dilation and misalignment in relation to the left ventricle. Furthermore, $N k \times 2-5^{183 P /+}$ mice also displayed increased trabecular layers on the left ventricular wall, indicative of left ventricular noncompaction, commonly observed in patients with $N K X 2-5$ mutations and also found in our patient cohort (22) (Figure 4B and Supplemental Videos 1-3). Functional analysis of hearts showed a highly significant right ventricular dysfunction in both mutant lines, associated with a significant decrease in right ventricle ejection fraction (Figure 4C and Supplemental Table 3). By contrast, left ventricular function was only affected in $N k \times 2-5^{183 M /+}$ mice, which showed decreased end diastolic volume (LV-EDV) and stroke volume (LV-SV) and heart mass (LV-mass) (Supplemental Table 3). The right ventricular dysfunction could be either a primary defect of the muscle or secondary to hemodynamic changes associated with the pulmonary circulation, as $N k \times 2-5$ is important for proper outflow tract formation during embryonic development $(31,32)$. To address whether right ventricular dysfunction in our mutants is due to changes in the pulmonary circulation, we analyzed pulmonary and aortic artery diameters using microCT in embryos 18.5-day after coitum, when the pulmonary circulation is still not functional. $N k \times 2-5^{183 P /+}$ embryos showed a significant increase in pulmonary artery diameter when compared with WT littermates (Supplemental Figure 3). In contrast, no changes in aorta diameter were observed (Supplemental Figure 3). Pulmonary artery enlargement would likely decrease pulmonary afterload and should not contribute to the right ventricular dilation, thus demonstrating that the defects seen in $N k \times 2-5$ mutants are likely 
A
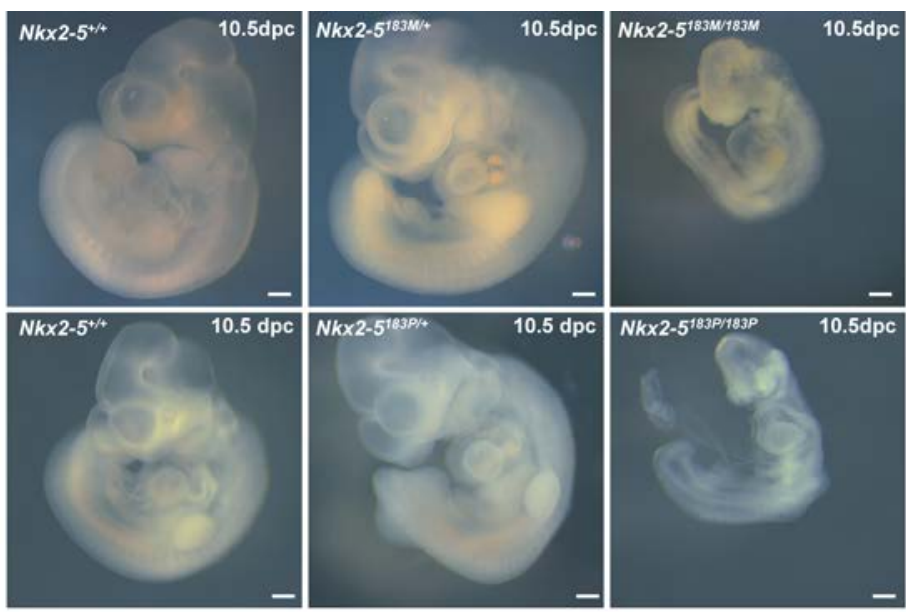

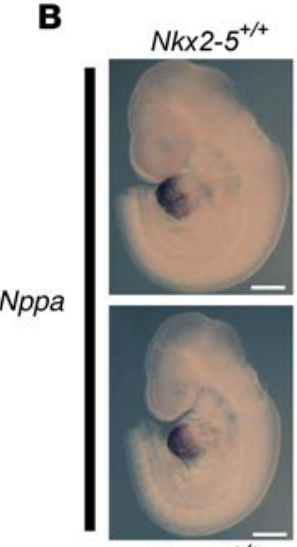

$N k \times 2-5^{+/+}$
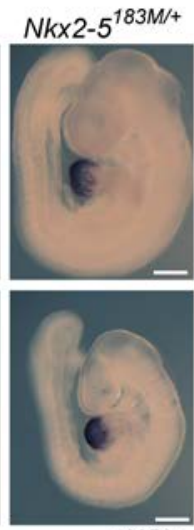

$N k \times 2-5^{183 P /+}$

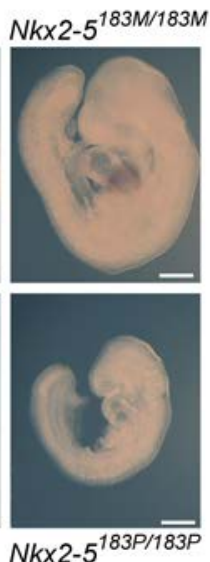

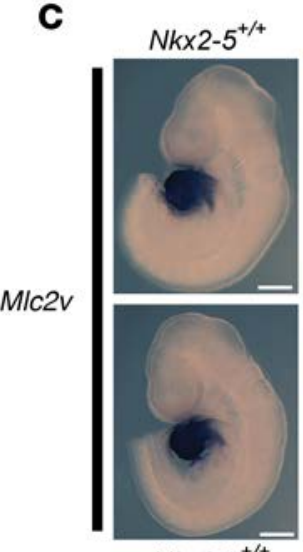

$N k \times 2-5^{+/+}$

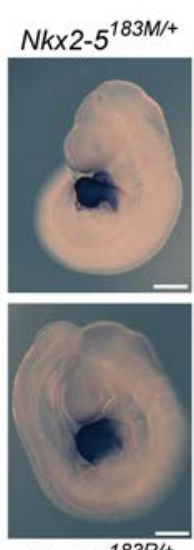

$N k \times 2-5^{183 M / 183 M}$

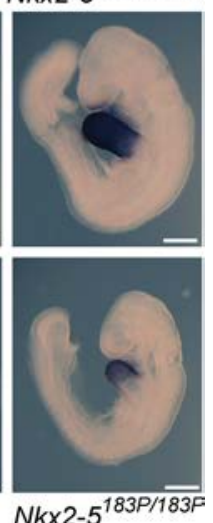

Figure 3. Homozygous $\mathbf{N k \times 2 - 5}$ point mutant mice are embryonic lethal at 10.5 days after coitum. (A) At 10.5 days after coitum, $N k \times 2-5^{183 M / 183 M}$ and $N k \times 2-5^{183 P / 183 P}$ homozygous embryos show severe growth retardation, failure in cardiac looping, and hypoplastic hearts. Nkx2-5+/+ and heterozygous mice develop normally and are born at Mendelian ratios. Scale bars: $250 \mu \mathrm{m}$. (B and C) In situ analysis of embryos 9.5 days after coitum shows decreased levels of Nppa expression (direct target of NKX2-5) in homozygous embryos, being more severe in NkX2-5838/1783P, and preserved expression of Mlc2v (a marker of cardiac specification). At least 4 embryos were used for each genotype. Scale bars: $200 \mu \mathrm{m}$. Nppa, natriuretic peptide A; Mlc2v, myosin light chain isoform $2 \mathrm{v}$.

primary to intrinsic muscle dysfunction. Together, these data indicate that mutations in the murine $N k \times 2-5$ gene cause severe right ventricular dysfunction in young adult animals, while compensatory mechanisms are most likely at place to ensure proper left ventricular function.

Presence of atrial septal defects in Nkx2-5 heterozygous mutant mice. One of the consistently observed phenotypes in patients with mutations in NKX2-5 is the presence of septal defects, accounting for $8 \%-19 \%$ of all detected familial atrial septal defects $(16,33,34)$. Atrial septum formation requires highly coordinated events that include directional growth, septa alignment, and apoptosis (24). During embryonic life, a direct route between right and left atria is maintained, allowing a bypass of the pulmonary circulation, which is replaced by the placenta in fetal development. After birth, this communication normally ceases to exist due to occlusion of the foramen ovale, caused by the increase of pressure in the left ventricle, which leads to fusion of the septum secundum to the foramen ovale; this allows the redirection of blood to the pulmonary circulation. This communication can, however, remain open in $15 \%-30 \%$ of individuals as a benign condition named patent foramen ovale.

Analysis of septum morphology showed an increased prevalence of patent foramen ovales in $N k \times 2-5$ heterozygous mice $\left(71 \%\right.$ in $N k x 2-5^{183 M /+}$ and $88 \%$ in $N k \times 2-5^{183 P /+}$ ) when compared with control mice (Nkx2-5/+ or WT littermates) (Table 1). As previously described for $N k \times 2-5^{4 /+}$ mice in a C57Bl/6J genetic background (24), we also detected marked enlargement of patent foramen ovales in $N k \times 2-5$ heterozygous mutants, with increased severity in 
A

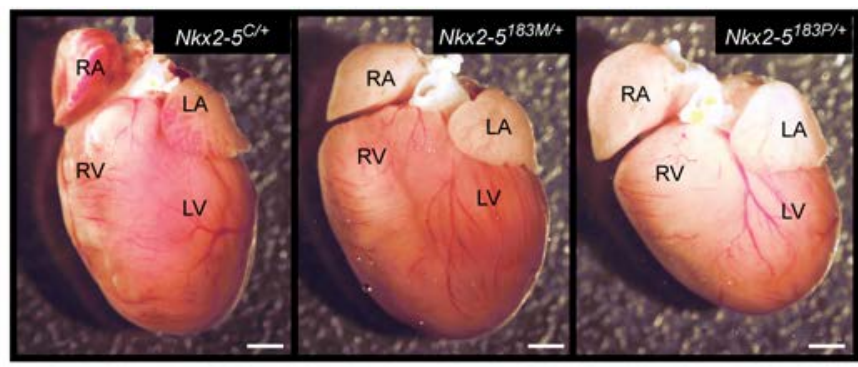

B

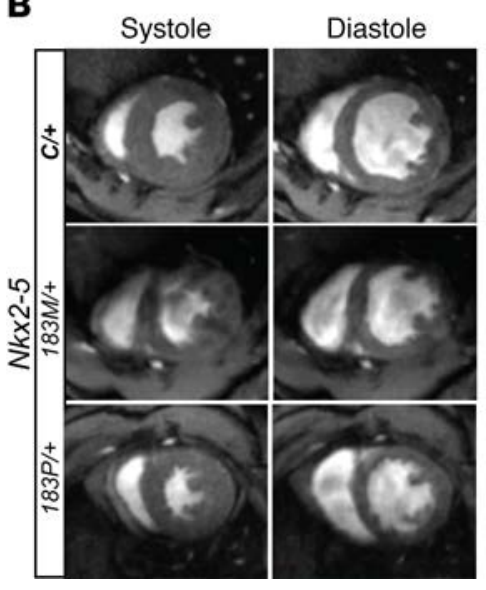

c
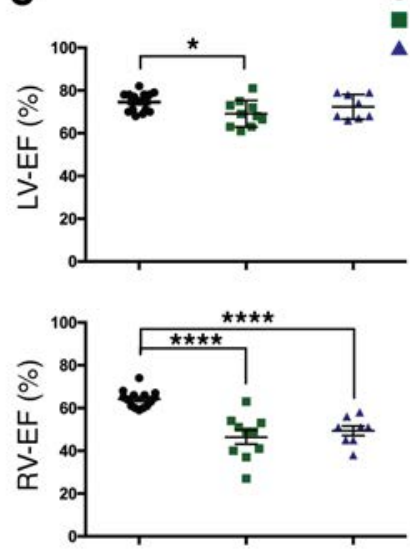

Figure 4. Heterozygous Nkx2-5 mice develop severe right ventricular dysfunction associated with mild left ventricular changes. (A) Hearts from 15-week-old mice display right ventricular dysmorphism with a shift to the base, rounded apex, and severe right atrial dilation when compared with $N k \times 2-5^{c /+}$ hearts. (B) Representative images from MRI show increased trabeculation or noncompaction of the left ventricle in Nkx2$5^{183 \mathrm{M} /+}$ with even stronger penetrance in Nkx2$5^{183 P /+}$ hearts. (C) Mild left ventricular dysfunction associated with a high degree of right ventricular dysfunction. Control: $n=14$; 183M: $n=10$; 183P: $n=$ 8. ${ }^{*} P<0.05$; ${ }^{* * *} P<0.0001$, unpaired $t$ test equal variance 2 tailed. RA, right atrium; LA, left atrium; $\mathrm{RV}$, right ventricle; $\mathrm{LV}$, left ventricle; $L V-E F$, left ventricular ejection fraction; RV-EF, right ventricular ejection fraction.

Nkx2-5 $5^{183 P /+}$ mice (Table 1). These large patent foramen ovales also showed altered morphology. Furthermore, we detected a high percentage of atrial septal defects $(12 \%)$ in Nkx2-5 $5^{183 P /+}$ adult mutant mice, not previously seen in either $N k \times 2-5^{4 /+}, N k \times 2-5^{C /+}$, or $N k \times 2-5^{183 M /+}$ lines $(24,29)$, indicating increased severity of septal abnormalities caused by proline mutation. The presence of atrial septal defects and increased prevalence of patent foramen ovales are in line with expected pathological outcomes observed in most patients showing mutations in NKX2-5 (30).

Nkx2-5 mutants display conduction defects, arrhythmia, and dysmorphic atrioventricular node. Defects in electric conduction are detected in most mouse models that show decreased Nkx2-5 activity, especially when this gene is deleted after neonatal stages (9-12). In these models, NKX2-5 activity was essential for proper development and homeostasis of the sinoatrial and atrioventricular nodes, as previous full conditional deletion of Nkx2-5 led to arrhythmic episodes and heart failure (10). NKX2-5 has also been proven essential for transcriptional regulation of several cardiac ion channels and receptors that play crucial roles in the generation and regulation of the action potential in the heart. In humans, mutations in $N K X 2-5$ are normally associated with electrical abnormalities and atrioventricular blocks $(5,29)$.

We observed electrocardiographic changes in some $N k \times 2-5^{183 M /+}$ mice ( 2 of 6 ) under homeostatic, nonanesthetized conditions in several electric intervals, including increased PQ and QRS, and a small tendency for decreased ST intervals in both lines (Figure 5A). Analysis of heterozygous Nkx2-5 mice using telemetry showed that $N k \times 2-5^{183 M /+}$ mutants displayed occasional arrhythmic episodes with highly irregular PR intervals, characteristic of the presence of ectopic sinoatrial foci leading to a wandering atrial pacemaker (4/5), associated with severe arrhythmia, which is typical of second-degree atrioventricular block (Figure 5B and Supplemental Figure 4). Nkx2-5183P/+ displayed possible split QRS waves (3/8), indicative of bundle branch heart block (Figure 5B and Supplemental Figure 4). Histological analysis of the atrioventricular node region revealed reduction in node size (in $N k \times 2-5^{183 M /+}$ mice, $n=4$ ) or highly dysmorphic appearance (in $N k \times 2-5^{183 P /+}$ mice, $n$ $=4$ ) (Figure 5C). Expression of direct targets of NKX2-5 (9, 29), such as ion channels Scn5a, Kcnh2, and Cacna1c that are essential for excitation-contraction coupling, were either unaltered or upregulated in

Table 1. Prevalence of atrial septal defect (ASD) and patent foramen ovale (PFO) in heterozygous mutant Nkx2-5 hearts

\begin{tabular}{lccc}
\hline Genotype & ASD & PFO & Notes \\
wT & $0 / 26(0 \%)$ & $10 / 26(38 \%)$ & \\
Nkx2-5 & $0 / 13(0 \%)$ & $5 / 13(38 \%)$ & \\
Nkx2-5 $^{183 M /+}$ & $0 / 21(0 \%)$ & $15 / 21(71 \%)$ & 1 with septal aneurysm \\
Nkx2-5 $^{183 P /+}$ & $2 / 17(12 \%)$ & $15 / 17(88 \%)$ & 6 with septal aneurysm
\end{tabular}


A
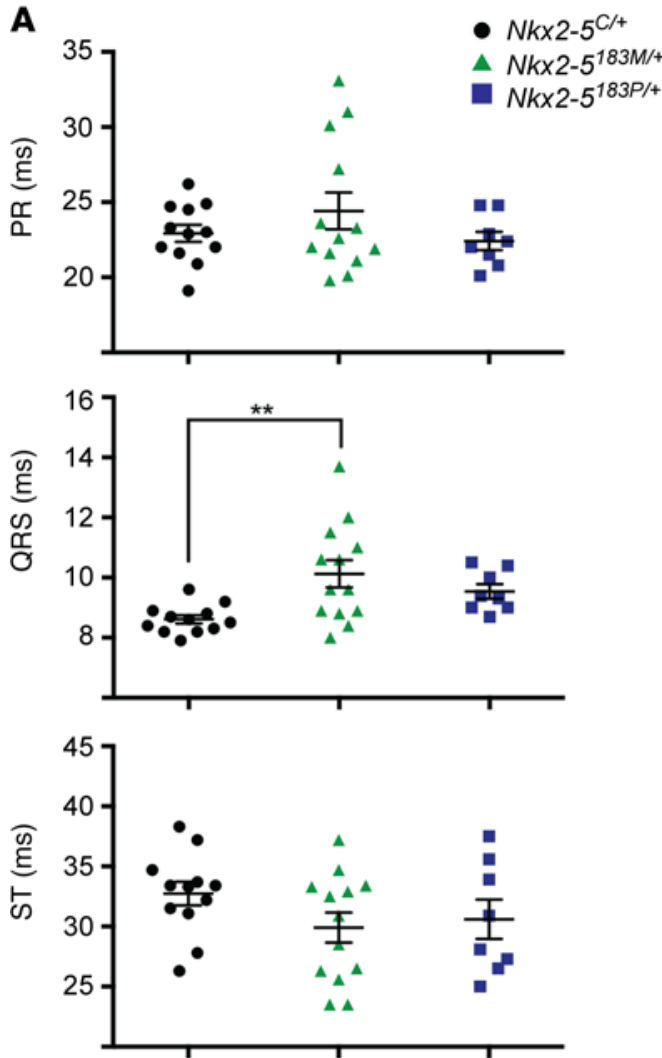

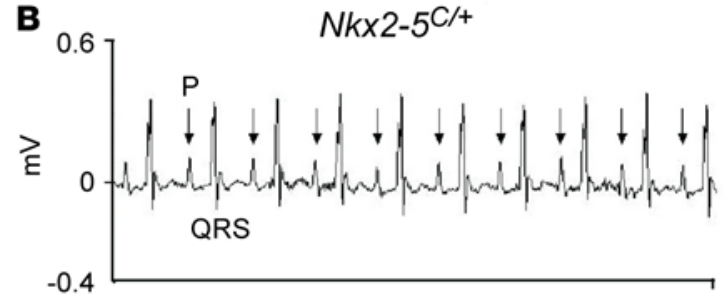

$N k \times 2-5^{183 M /+}$
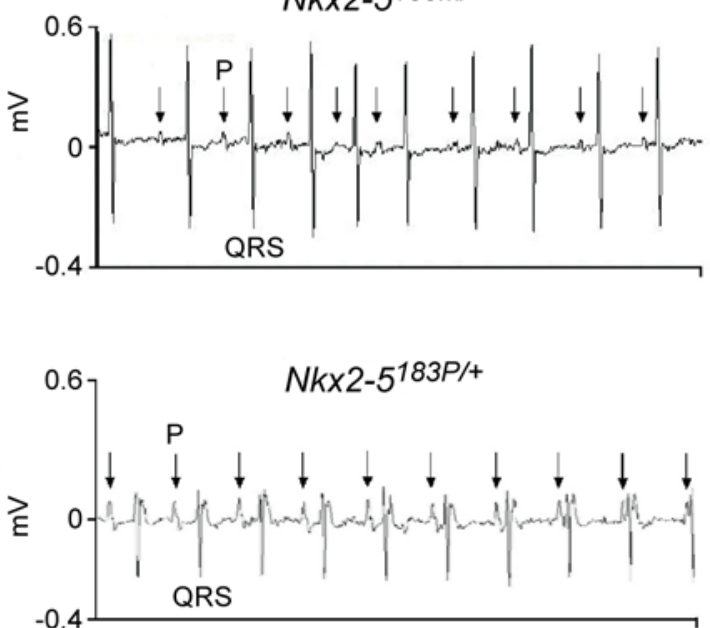

C

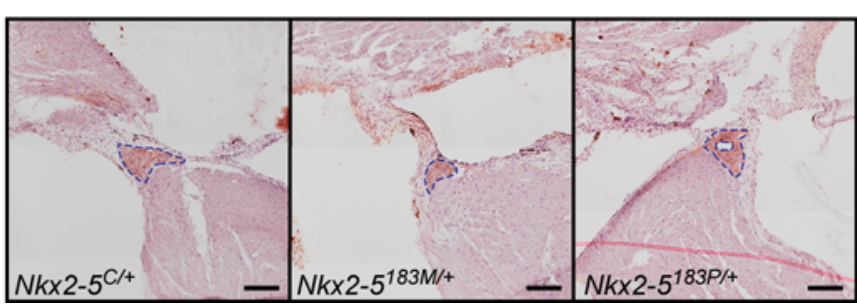

Figure 5. Electrical disturbances detected in heterozygous Nkx2-5 mice. (A and B). Nonanesthetized and telemetric electrocardiogram analyses show a wide variability on QRS intervals of $N k \times 2-5^{183 \mathrm{M} /+}$, caused by frequent asymmetric atrial pacemaking activity (wandering atrial pacemaking). Similar widening of ST intervals were also detected in both mutants, with $N k \times 2-5^{183 P /+}$ mice displaying frequent bundle blocks, widening of the QRS, and atrioventricular rhythm dissociation. In A, control: $n=12 ; 183 \mathrm{M}: n=13,183 \mathrm{P}: n=8$. In B, control: $n=5 ; 183 \mathrm{M}: n=5,183 \mathrm{P}: n=8$. (C) Abnormal atrioventricular node in heterozygous Nkx2-5 mutant mice, $n=3$ of each genotype. ${ }^{* *} P<0.01$, unpaired $t$ test equal variance 2 tailed. Scale bars: $200 \mu \mathrm{m}$. P, P wave; QRS, QRS complex; ST, ST wave interval.

mutant hearts, contrary to the expected downregulation (Supplemental Figure 5), suggesting that a compensatory mechanism is in place to sustain the essential role of excitation-contraction coupling in cardiac homeostasis, as previously observed for $N k \times 2-5^{4 /+}$ mice (29).

Mutations in NKX2-5 do not affect overall protein levels. We have previously shown increased stability of NKX2-5 mutant proteins I-M and I-P in vitro, an event associated with decreased ubiquitin proteasome system-mediated degradation (22). In order to test if this stability was maintained in vivo, we performed Western blots from adult hearts. Contrary to our previous in vitro observations (22), no significant changes in NKX2-5 levels were detected between control or heterozygous mutant hearts (Figure 6), indicating that, at least in vivo, compensatory mechanisms balance protein production and degradation to maintain normal NKX2-5 overall levels in adult hearts. Whether mutant proteins retain WT transcriptional activation capacity in vivo remains to be determined.

Molecular analysis identifies altered pathways in Nkx2-5 mutant hearts. NKX2-5 protein persists in the heart throughout adult life, where the presence of one mutant copy contributes to a wide variety of cardiac 


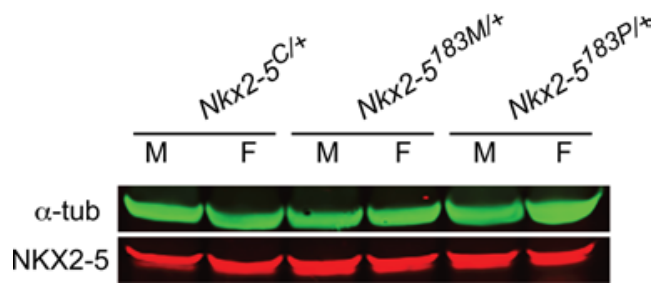

Figure 6. NKX2-5 protein levels are not affected in heterozygous mouse lines. Western blot analysis of NKX2-5 protein levels using 15 -week-old adult hearts. $\alpha$-Tubulin was used as endogenous loading control. $\alpha$-tub, $\alpha$-tubulin; M, male; F, female. phenotypes observed in patients (30). To dissect the long-term effects of mutated proteins, we performed microarray analyses of $N k \times 2-5^{183 M /+}, N k \times 2-5^{183 P /+}$, and $N k \times 2-5^{183 C /+}$ adult mutant hearts and the heterozygous null $N k \times 2-5^{4 /+}$ model, which does not display the phenotype observed in the knock-in models (29). Global analysis of these transcriptional profiles showed that several known direct targets of NKX2-5 in embryonic development were highly compensated in adult heterozygote hearts, such that few genes $(290$ of 55,821$)$ were differentially regulated between control and knock-in hearts (Figure 7A; 2-fold changes, $P<0.05$ ). The large majority of differentially expressed genes displayed modest 2- to 4-fold changes. In line with functional data, molecular profiling demonstrated a gradient of phenotype between $183 \mathrm{M}$ and $183 \mathrm{P}$ lines; $N k \times 2-5^{183 P /+}$ hearts showed a more pronounced change in gene expression with proportionally more repressed genes when compared with $N k \times 2-5^{183 M /+}$ hearts. A stringent cut off $(P<0.001)$ highlighted a subset of genes likely associated with the cardiac phenotype in $N k \times 2-5^{183 M /+}$ and $N k \times 2-5^{183 P /+}$ adult hearts (Figure 7B). Within the genes sets coregulated by both $N k \times 2-5^{183 M /+}$ and $N k \times 2-5^{183 P /+}$ hearts (Supplemental Table 4), genes associated with processes of cell adhesion (Scgb1c1), ion handling (Cacna2d1), proliferation/apoptosis (Ndrg4), cell signaling, organ morphogenesis, and homeostasis (Jmjd6, Six1, Scube, Myl1) were identified and further validated by qPCR (Figure 7C).

Interestingly, $N k \times 2-5^{4 /+}$ heart profiling showed a large degree of differential regulation compared with the point-mutants with 205 unique genes, while comparison of $N k \times 2-5^{183 M /+}$ and $N k \times 2-5^{183 P /+}$ hearts revealed only 41 and 48 uniquely regulated genes, respectively. Despite the larger transcriptional changes observed in the $N k \times 2-5^{4 /+}$ hearts, it is important to note that this line does not develop a phenotype consistent with the full presentation of the human disease, underscoring the importance of having the appropriate model for dissection of mechanistic information (Figure 7B and Supplemental Table 4).

Comparative analysis of all differentially regulated $N k \times 2-5^{183 M /+}$ and $N k \times 2-5^{183 P /+}$ targets with direct targets of DamID-WT NKX2-5 protein in HL-1 cells shows a low degree of correlation, with only 58 common targets (Supplemental Figure 6). These few common targets between HL-1 and our adult heart datasets are likely the direct targets of NKX2-5 affected in the adult onset disease. These findings also suggest that most altered transcripts in the adult hearts $(1,509$ of 1,567$)$ are secondary or off-targets of NKX2-5 (35).

Mitochondrial dysfunction in Nkx2-5 $5^{183 P /+}$ cardiomyocytes. Using Mitocarta 2.0 (36), we identified an extensive list of nuclear mitochondrial-associated genes that were differentially expressed in $N k \times 2-5^{183 P /+}$ hearts and postulated an involvement of a primary respiratory defect in the phenotype of point-mutations (Supplemental Table 5). To follow up on this issue, we used the Seahorse technology (Agilent) to perform realtime measurements of energy utilization in living cells and observed a severe impairment of mitochondrial function in neonatal cardiomyocytes derived from $\mathrm{Nkx} 2-5^{183 P /+}$ mutant hearts. While no significant changes in ATP production or proton leak were seen, a dramatic decrease was observed in basal and maximal respiration, associated with a much lower baseline respiratory capacity (Figure 8). This suggests that respiration levels are close to maximum capacity under homeostatic conditions in Nkx2-5 mutant cardiomyocytes, likely not allowing for any respiratory reserve, if required.

Nkx2-5 dysfunction leads to significant changes in Wnt signaling in adult hearts. Using ingenuity pathway analysis (IPA, Qiagen), we identified the process of cardiogenesis as the major affected canonical pathway in $N k \times 2-5^{183 P /+}$ hearts. The main altered genes in this process were the signaling pathways for BMP, TGF $\beta$, and Wnt (Supplemental Figure 7). Wnt signaling is essential for heart development, patterning, and adult homeostasis and adaptation (37). Several differentially regulated transcripts were detected in our analysis (Table 2) and validated by qPCR in $N k \times 2-5^{183 M /+}$ and $N k \times 2-5^{183 P /+}$ adult hearts (Figure 9A) and neonatal cardiomyocytes (Supplemental Figure 8). Transcripts for Fzd7, Tnik, Rock2, and Myl1 showed increased levels in $N k x 2-5^{183 M /+}$ and $N k \times 2-5^{183 P /+}$ mutants compared with WT samples, while Hopx levels were downregulated in $N k \times 2-5^{183 P /+}$ mice. Interestingly, Hopx has been recently shown to be an essential repressor of the Wnt signaling that promotes cardiomyogenesis (38). Furthermore, $N k \times 2-5^{183 P /+}$ neonate cardiomyocytes displayed sensitivity to increased concentrations of Wnt3a, measured by increased accumulation of $\beta$-catenin on the cell surface membrane when compared with WT cardiomyocytes (Figure 9B). Hypersensitivity to Wnt signaling was further confirmed in transient transfection assays using the $\beta$-catenin-dependent Wnt reporter TopFlash. Using the ligand Wnt3a as a surrogate for canonical Wnt pathway activation, both $N k \times 2-5^{183 P /+}$ and control neonatal cardiomyocytes were capable of responding to increasing dosages of Wnt (Figure 9C), although $N k \times 2-5^{183 P /+}$ cardiomyocytes showed significantly higher activation of TopFlash when compared 
A

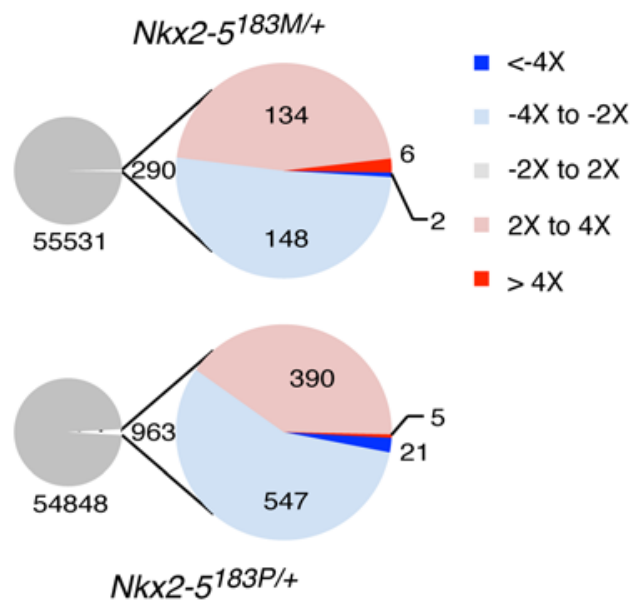

B

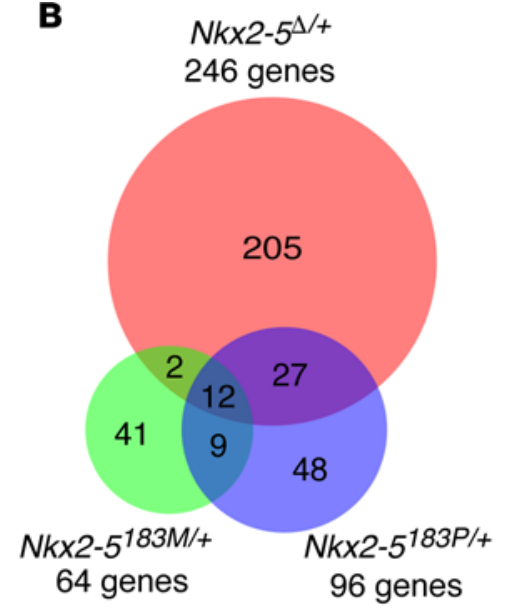

C
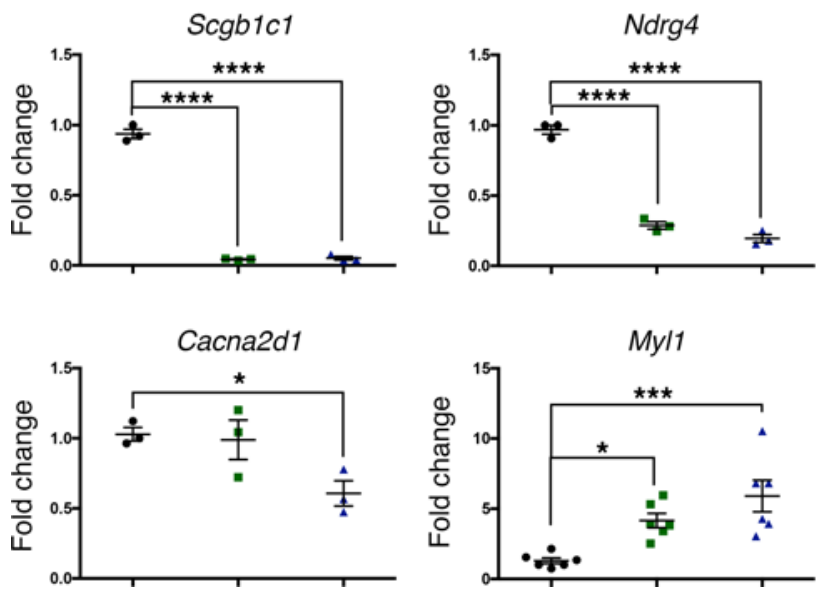
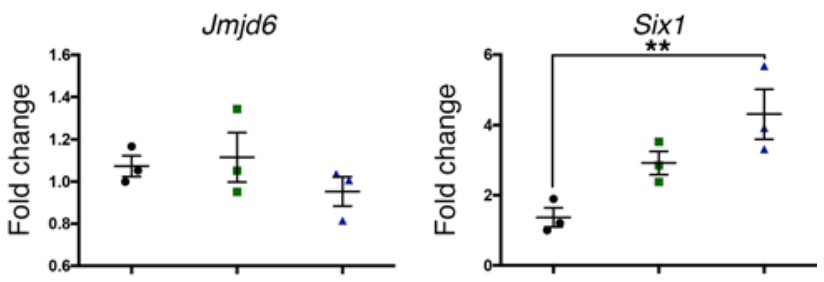

- Contro

Nkx2-5 $183 M /+$

A $N k \times 2-5^{183 P /+}$

Figure 7. Transcriptome changes in adult heterozygous $\mathbf{N k x 2 - 5}$ mouse hearts. (A) Global analysis shows subtle changes in the transcriptional profile between adult heterozygous mice and controls $(P<0.05)$. Color scheme reflects differential levels of expression, with lowest in gray, highest upregulation in deep red, and highest downregulation in deep blue. (B) Venn diagram comparing gene changes in both $N k \times 2-5^{4 /+}$ and $N k \times 2-5^{183 M /+}$, and $N k \times 2-5^{183 P /+}$ in relation to $N k \times 2-5^{C /+}$ mice show few common genes among all models. The most significant changes are seen for $N k \times 2-5^{4 /+}$, which does not phenocopy the human disease $(P<0.001)$. (C) qPCR validation of main differentially expressed targets shown as fold change normalized by expression of $H p r t 1 .{ }^{*} P<0.05$; ${ }^{* *} P<0.01 ;{ }^{* *} P<0.001 ;{ }^{* * *} P<0.0001,1$-way ANOVA.

to control cardiomyocytes. Collectively, these results indicate enhanced sensitivity of the response to Wnt signaling in Nkx2-5 mutant neonatal cardiomyocytes that could predispose them to the observed non-compaction phenotype in adult mutant hearts (Figure 4B and Supplemental Videos 1-3).

Besides its well-established role during cardiac development and specification, Wnt is essential in modulating cardiac response to injury by myocardial infarct (MI), hypertrophy and hypoxia/reperfusion (H/R) (37), and we observed an increase in Wnt3a levels in WT neonatal cardiomyocytes exposed to H/R or hearts subjected to MI (Figure 10A). Addition of Wnt3a also led to a substantial decrease in mitochondrial membrane potential (Figure 10B) and metabolism in neonatal cardiomyocytes (Supplemental Figure 9). In contrast, cardiomyocytes isolated from $N k \times 2-5^{183 P /+}$ mice showed the opposite response in basal respiration and ATP production, and they were not capable of increasing respiratory capacity in response to Wnt3a (Figure 10C). This indicates that, in normal cardiomyocytes, upregulation of Wnt signaling limits cardiomyocyte respiratory activity, which decreases maximal respiratory capacity and increases reserve. $N k \times 2-5^{183 P /+}$ cardiomyocytes, on the other hand, utilize their respiration to their maximum capacity at all times and are not capable of responding to the Wnt3a stimulus. These findings strongly implicate Wnt in Nkx2-5-mediated control of cardiomyocyte metabolism. 


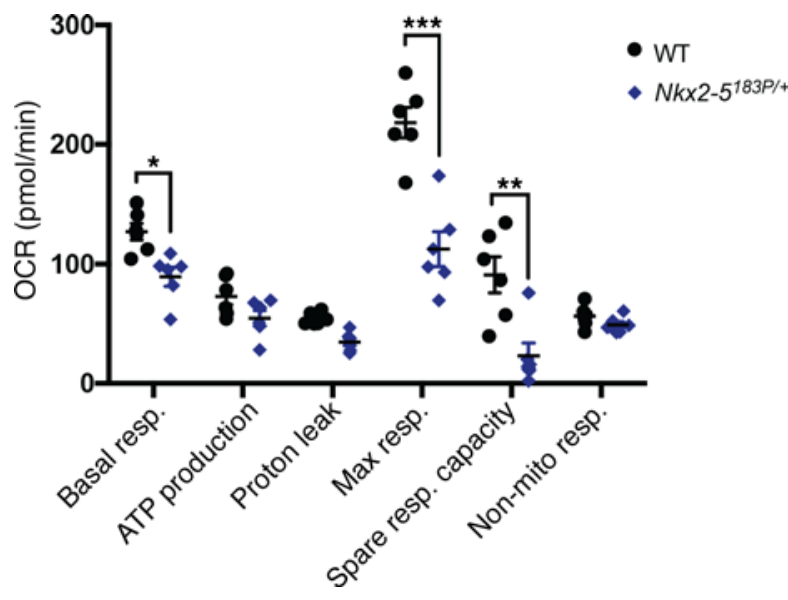

Figure 8. Functional changes are associated with decreased mitochondrial respiratory activity in neonatal cardiomyocytes. $\mathrm{Nkx2-5183P/+}$ neonatal cardiomyocytes mitochondrial activity measured as basal respiration, ATP production, proton leak, maximal respiration, spare respiratory capacity, and nonmitochondrial respiration. $(n=5){ }^{*} P<0.05$; ${ }^{* *} P<0.01$; ${ }^{* *} P<0.001$, unpaired $t$ test equal variance 2 tailed. OCR, oxygen consumption rate.

\section{Discussion}

The accelerated discovery of genetic mutations associated with human cardiac disease calls for the establishment of precise models to study the relationship between genetic predisposition and cardiac dysfunction. Previous mouse models carrying homozygous or heterozygous Nkx2-5 gene deletions (9-12) displayed some but not all phenotypic characteristics of the human disease. Although phenotype discrepancies in these models could be interpreted as differences in experimental approaches and/or depth of analyses, clinical manifestations of NKX2-5 dysfunction are likely caused by the interaction between WT and mutated proteins, as is the case in our patient cohort (22), and not by full lack of a gene allele. Transcription factors operate in highly organized multimeric complexes with cofactors and general transcriptional machinery factors ( 35 , 39-42). Few examples exist in the literature where heterozygous deletion of alleles reflects the human disease, as seen in Tbx5 and Holt-Oram Syndrome models (43).

In the case of $N K X 2-5$, most human cardiac defects are associated with heterozygous missense mutations concentrated in the conserved homeodomain region, which cause impairment of transcriptional activity mediated by decreased DNA binding (5). The knock-in models presented here are therefore ideal for the study of the molecular pathways altered by long-term heterozygous mutant protein expression. In heterozygous configuration, they faithfully reproduce the major clinical characteristics of congenital heart disease associated with mutations in this gene in patients, including atrial septal defects, conduction defects, and noncompacted myocardium. The embryonic lethality observed in homozygous I-M and I-P point mutants, similar to that observed in $N k \times 2-5$ deletion $(7,8,29)$, illustrates the strong disruption of the cardiogenic program, even in the presence of an otherwise intact NKX2-5 protein, underscoring the critical importance of this residue in protein function.

Genetic background presents a further variable for consideration when building mouse models. The more penetrant cardiac phenotype of an Nkx2-5 R189G knock-in compared with the clinical presentation seen in patients was strongly dependent on the genetic background used for the mouse studies, with highest severity associated with 129/SV and diluted in a mixed 129/SV;C57B16 background (44). In the present study, replacement of the NKX2-5 isoleucine residue by methionine, the actual human mutation, produced a less severe phenotype than proline, a more severe but artificial change, which may also be affected by genetic background. In general, penetrance of phenotype is more dependent on genetic modifiers in inbred mice than in humans $(24,45-47)$.

In spite of the observed primary cardiac dysfunction of the right side of the heart at both morphological, contractile, and cellular respiratory levels, young adult Nkx2-5 knock-in mice still displayed a high degree of physiological compensation that allowed for normal function, as illustrated by left ventricular ejection fraction. To our knowledge, right ventricular dysfunction in patients has so far not been associated with mutations in Nkx2-5, and it is a rather understudied phenomenon. A more detailed analysis of right ventricular dysfunction should be performed in patients that carry $N K X 2-5$ mutations, given the role $N k \times 2$ 5 plays in outflow tract and pharyngeal arch formation, as well as the increased interest and importance of the RV cardiac function in heart failure $(32,48,49)$. Intriguingly, $N k \times 2-5^{183 M /+}$ hearts were significantly smaller than both $N k \times 2-5^{183 C /+}$ and $N k \times 2-5^{183 P /+}$ hearts. It remains to be addressed whether this mutation has particular implications for determination of heart size, an unknown association with $N k \times 2-5$ gene function.

Our model also reproduced septal defects normally associated with NKX2-5 mutations in patients. Previous studies in adult $N k \times 2-5$ mutant mice showed increases in atrial septal defects only in early neonatal stages $(46,47)$, raising the possibility that atrial septal defects could resolve with cardiac maturation after birth. Others reported septation defects that were much more severe than seen in patients (44). Our results are more consistent with the human condition, with atrial septal defects persisting in adult mice $\left(N k \times 2-5^{183 P /+}\right)$. Another common observation among Nkx2-5 knock-in models (50) is the presence of electrophysiological abnormal- 
Table 2. Differential expression of Wnt pathway genes in adult $N k \times 2-5$ mutant hearts

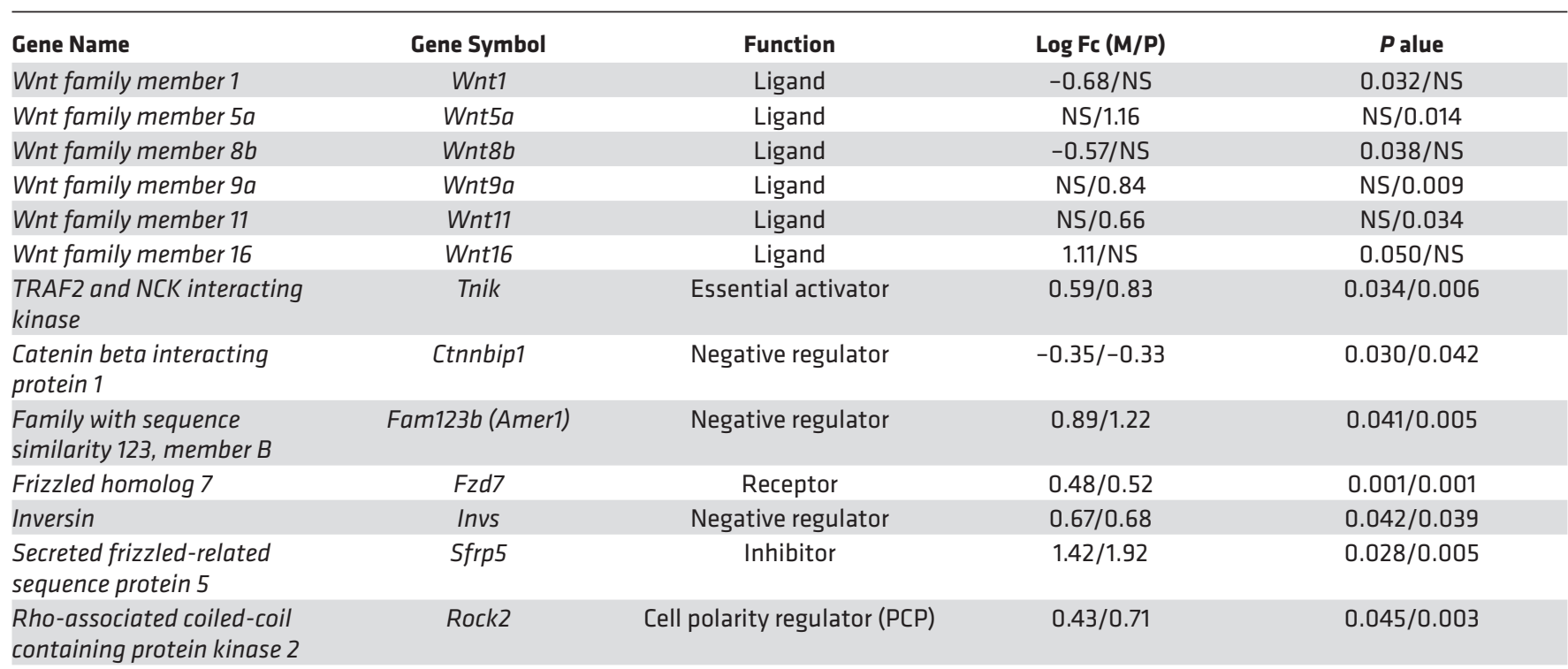

ities. In addition to dysmorphic atrioventricular nodes, the $N k \times 2-5^{183 M /+}$ line also displayed severe arrhythmic events characterized by wandering atrial pacemaker most likely caused by extra atrial pacemaking activity, perhaps associated with the severe right atrial dilation. In contrast, $N k \times 2-5^{183 P /+}$ mice occasionally displayed complete atrioventricular block, associated with atrioventricular dissociation and possible bundle block.

Molecular analysis of adult Nkx2-5 mutants showed an unexpectedly high degree of compensation at the mRNA level, as proven by the small degree of transcriptional changes in $N k \times 2-5$ heterozygous hearts when compared with controls. Among the small proportion of NKX2-5 direct targets affected in the knock-in lines (Table 2 and Supplemental Figure 7), several genes associated with the Wnt signaling pathway were of particular interest, as Wnt signaling has been associated with a wide range of developmental processes, including cellular specification, tissue patterning, and proliferation. During heart development, canonical and noncanonical Wnt signaling have temporally distinct roles, with an initial inductive role for cardiac specification followed by a repressive activity that promotes cardiomyocyte differentiation at later stages (51). Neonatal cardiomyocytes express several components of the Wnt pathway (52) that are subsequently silenced in the adult heart. Although this pathway is reactivated in response to cardiac injury, repression is required for adaptive hypertrophy (53-55). Wnt3a signaling has been indirectly linked to Nkx2-5 through the regulation of Scna5 channel, which is a direct target of NKX2-5 (52). NKX2-5 has been shown to directly repress $\beta$-catenin expression in ventricular myocytes (56). We did not detect changes in mRNA levels for $\beta$-catenin in our model. Contrary to experiments performed by Riazi et al., where Nkx2-5 was knocked down using an antisense strategy, our model is a heterozygous system where one WT copy and a mutant copy are present (56). In addition, large changes in $\beta$-catenin expression levels are not required to promote Wnt signaling, but rather changes in phosphorylation status of presynthesized protein associated with increase of other pathway components, such as receptors and regulatory proteins, can exert a similar modulatory effect of the Wnt pathway (37).

A role for Wnt signaling in Nkx2-5 mutant phenotypes was also supported by a significant decrease in Hopx transcripts in Nkx2-5 mutant neonatal cardiomyocytes (Figure 9A). Hopx has been implicated in cardiomyocyte specification through its repression of Wnt signaling, failure of which leads to noncompaction (38). A clear left ventricular noncompaction phenotype, with increased trabeculation and deep intratrabecular recesses, was especially evident in $N \times 2-5^{183 P /+}$ hearts, similar to observations in patients (57).

The primary dysfunction of mitochondrial metabolism found in this study (Figure 8, Supplemental Table 5, and Supplemental Figure 9) is consistent with the observed cardiac defects, including left 

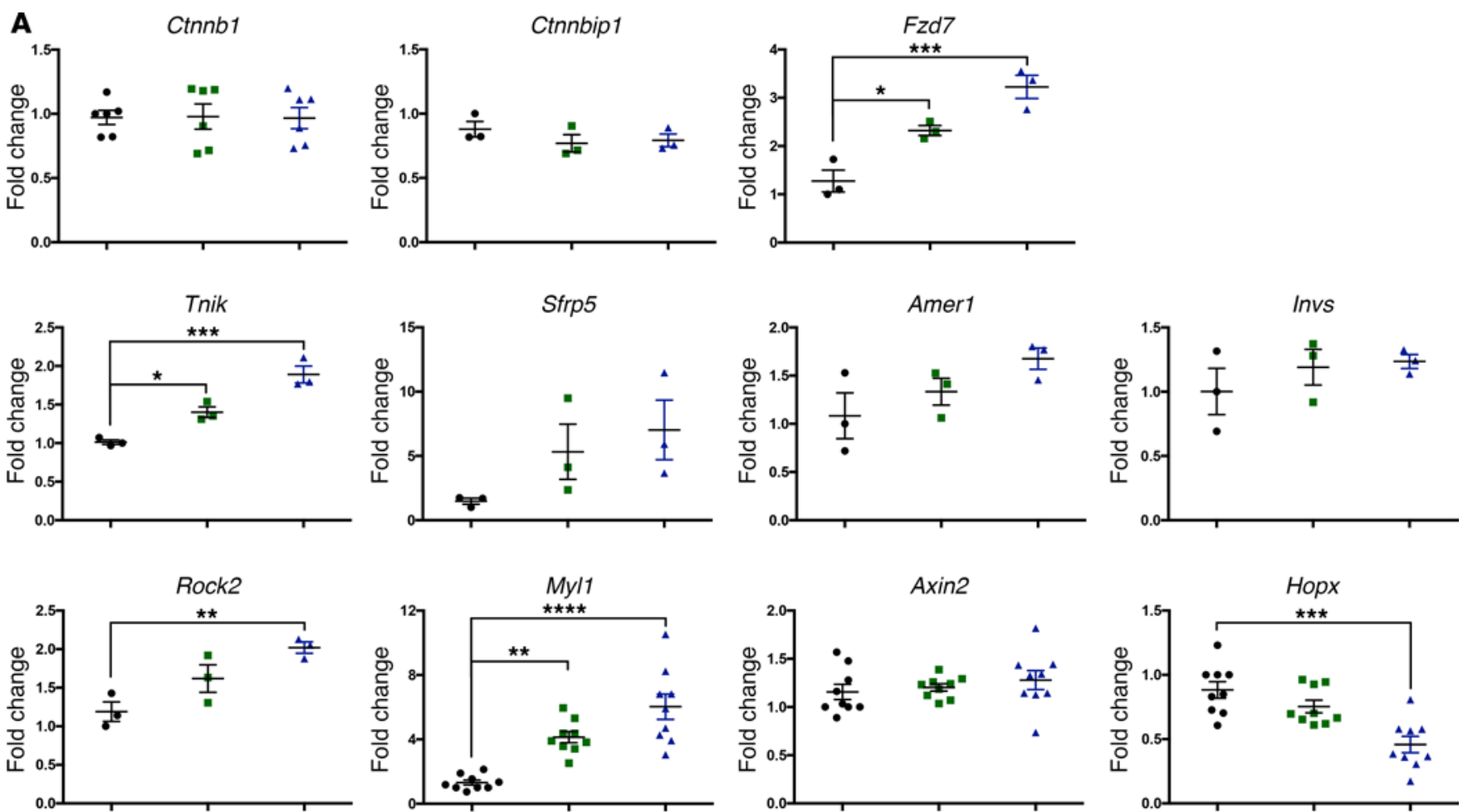

B

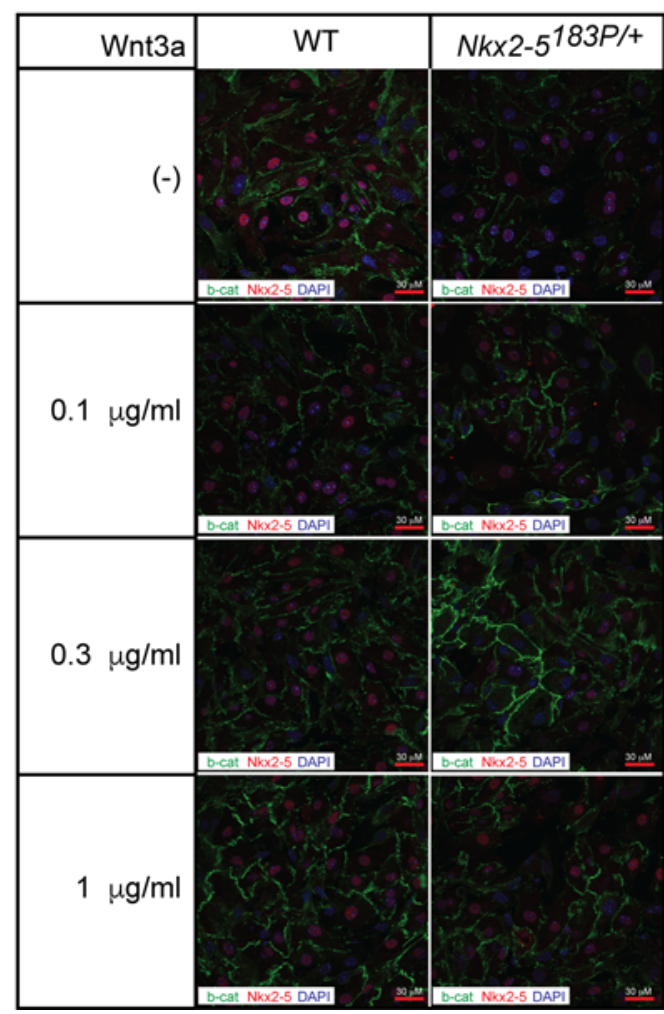

C

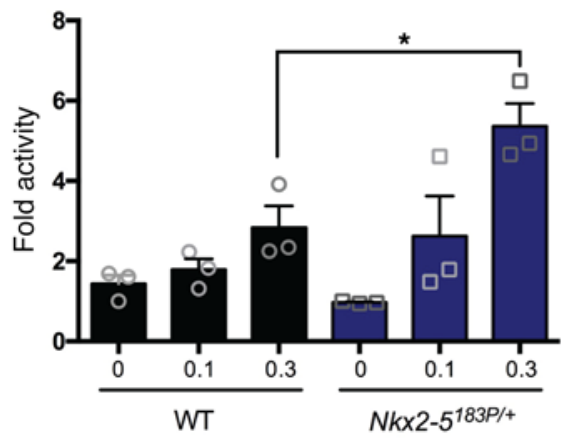

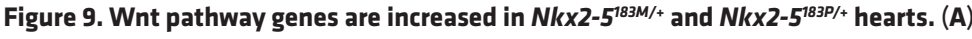
qPCR targets associated with the Wnt pathway in adult hearts, shown as fold change over Hprt1. (B) Increased Wnt3a sensitivity reported by accumulation of $\beta$-catenin (b-cat, green) in the membrane of $\mathrm{Nk} \times 2-5^{183 P /+}$ neonatal cardiomyocytes. (C) Dose-dependent activation of $\beta$-catenin-dependent TopFlash reporter construct from control (WT) and Nkx2-583P/+ mutant neonatal cardiomyocytes, expressed as fold activity of luciferase readings over unstimulated cells (no Wnt), normalized by Renilla expression. Wnt concentrations shown in $\mu \mathrm{g} / \mathrm{ml} . n=3 .{ }^{*} P<0.05 ;{ }^{* *} P<0.01 ;{ }^{* * *} P<0.001$; ${ }^{* * * *} P<0.0001,1$-way ANOVA (A) and unpaired $t$ test equal variance 2 tailed (C).

ventricular noncompaction $(58,59)$. Furthermore, Hopx also directly controls Bmp signaling through interaction with activated Smads, thereby influencing Wnt-Bmp pathway regulation of cardiomyocyte specification (38). In fact, concomitant changes in Bmp-associated molecules were also seen in our model, including downregulation of Bmp1/5/7/10 and Smad1 and upregulation of Smads5/6 (Supplemental Figure 7 and Supplemental Table 6). A similar left ventricular noncompaction phenotype upon 
A

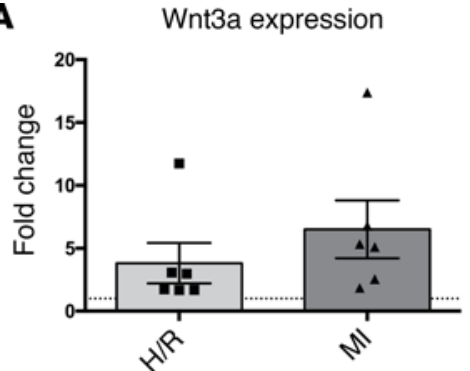

B

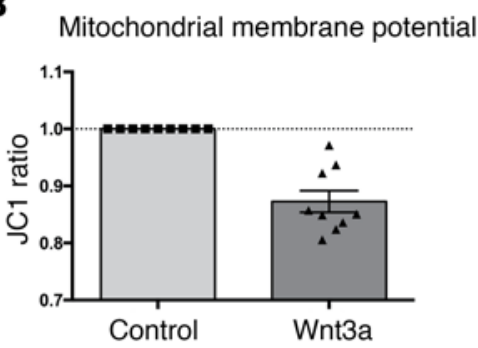

\section{C}

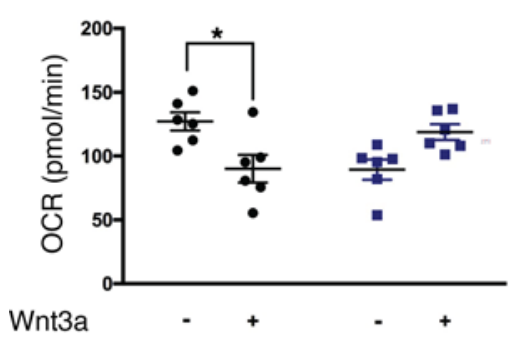

Max respiration

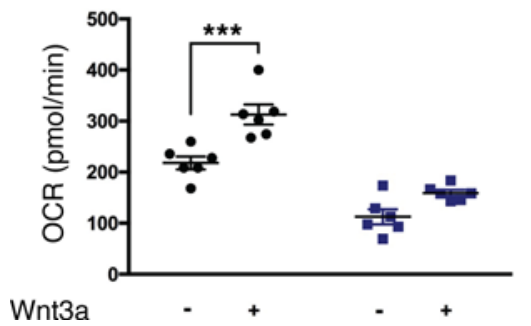

ATP production
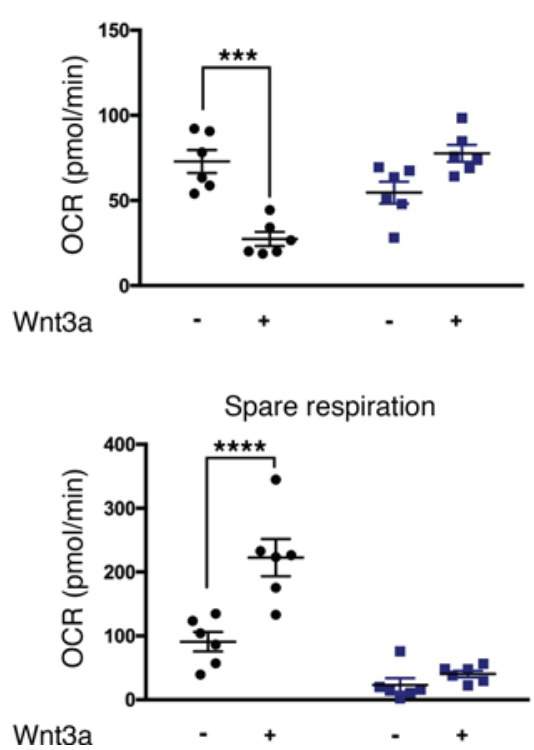

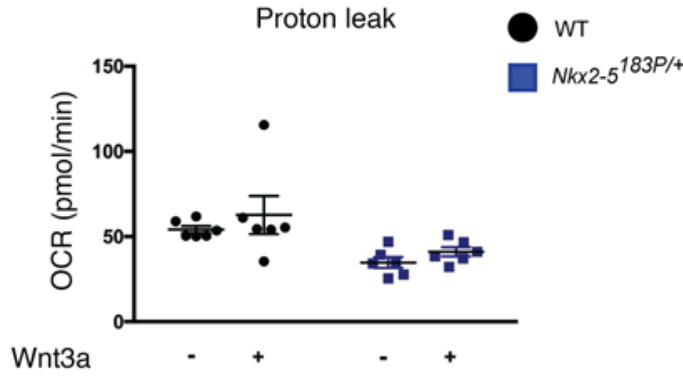

Non-mitochondrial respiration

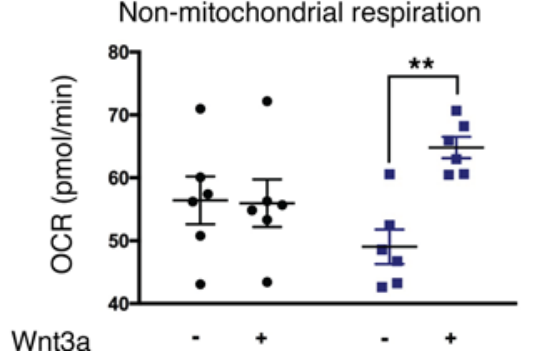

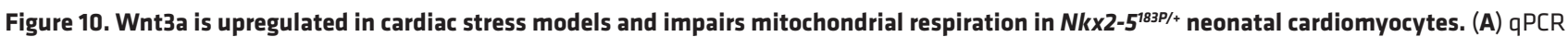
shows an increase in Wnt3a expression in models of cardiac injury through hypoxia/reperfusion or myocardial infarction. Line represents control levels of Wnt3a in normoxic (hypoxia/reperfusion) and sham operated (myocardial infarction) mice. Results are shown as fold changes normalized by Hprt1 expression. (B) Supplementation of Wnt3a to primary neonatal cardiomyocytes leads to decreased mitochondrial membrane potential $(n=6)$, measured by JC-1 membrane permeant dye staining. (C) WT cardiomyocytes show better mitochondrial performance in all measured parameters when compared with Nkx2-5 ${ }^{183 P /+}$ (basal respiration, ATP production, proton leak, maximum respiration, and spare respiration). Upon Wnt3a stimulation, WT cardiomyocytes reduce energy consumption and increase spare respiratory capacity, while $\mathrm{Nk} \times 2-5^{183 P /+}$ cardiomyocytes retain similar low levels of mytochondrial activity and dramatically increase nonmitochondrial respiration. $n=6$. ${ }^{*} P<0.05 ;{ }^{* *} P<0.01 ;{ }^{* *} P<0.001$; ${ }^{* * *} P<0.0001$, unpaired $t$ test equal variance 2 tailed. $\mathrm{MI}$, myocardial infarction; H/R, hypoxia/reperfusion; OCR, oxygen consumption rate in picomols per minute.

Nkx2-5 deletion in adult ventricular cardiomyocytes was associated with upregulation of Bmp10 (10). These results are consistent with the NKX2-5 control of the Hopx-Wnt-Bmp axis. Thus, disturbances in the Wnt pathway may be at the core of morphological changes observed in $N k \times 2-5$ mutant mice.

Contrary to previous reports where NKX2-5 can bind and directly regulate expression of $\beta$-catenin gene (Ctnnb1) (56), no changes were seen in expression of Ctnnb1 in our model. Axin2, a direct target of $\mathrm{Wnt} / \beta$-catenin activation, was also unchanged, reinforcing the existence of a large degree of transcriptional compensation in adult hearts. We have, however, seen increased potentiality of $N k \times 2-5^{183 P /+}$ cardiomyocytes to respond to a $\beta$-catenin element (TopFlash) once stimulated with Wnt3a, consistent with the upregulation of several genes of the pathway in $N k \times 2-5^{183 P /+}$ hearts, including the overexpression of the Wnt receptor $F z d 7$. At functional level, point-mutant $N k x 2-5^{183 P /+}$ cardiomyocytes show impaired mitochondrial respiratory capacity in homeostasis, similar to what is observed upon Wnt3a activation in stress for WT cardiomyocytes. Addition of exogenous Wnt3a fails to reduce this response even further, implying that $N k \times 2-5^{183 P /+}$ cardiomyocytes are insensitive to enhancement of this pathway. Taken together, our data suggest that $N k \times 2-5^{183 P /+}$ cardiomyocytes show increased Wnt signaling in homeostatic conditions, likely leading to the observed respiratory dysfunction. We predict that these events will lead to decreased cardiac capacity, energetic impairment, and worsening of cardiac disease. 
One of the biggest challenges for the understanding of complex congenital heart disease lies on the large number of genes involved and the high phenotypic variability observed (60). The emergence of proper disease mammalian models is therefore paramount in the dissection of processes and pathways associated with adult congenital heart disease, such as the ones described here for Nkx2-5. These knockin models will be invaluable in enhancing the analysis of environmental events and modifiers of cardiac function (e.g., obesity, high blood pressure) in a context of genetic predisposition, an increasing consequence of high survival rates associated with clinical interventions in pediatric cardiology.

\section{Methods}

Mouse lines generation. $\mathrm{Nkx} 2-5^{\mathrm{C} /+}, \mathrm{Nkx} 2-5^{183 \mathrm{M} /+}$, and $\mathrm{Nkx} 2-5^{183 \mathrm{P} /+}$ mice were generated by the Gene Recombineering and Embryonic Stem Cell Targeting Facilities at the Australian Regenerative Medicine Institute, Monash University. Neomycin cassette deleted lines (Nkx2-5C $\Delta, \mathrm{Nkx} 2-5 \mathrm{M} \Delta$ and Nkx2-5P 4 ) were obtained by crossing with the CMV-Cre deleter line (61). $N k x 2-5^{f /+}$ and $N k x 2-5^{4 /+}$ are described elsewhere (29). All mice were kept on a C57BL6/J background, housed at Monash Animal Services - Monash University or at the Rodent Animal Facility - the Jackson Laboratory.

Histology, immunofluorescence, and acetylcholinesterase (AchE) staining. Hearts were fixed in 4\% paraformaldehyde at $4^{\circ} \mathrm{C}$ for 12 hours and further processed for paraffin embedding and histological analysis. Sections were analyzed using Masson's Trichrome staining. For myocyte area measurements, transverse sections were stained with Wheat Germ Agglutinin-AF 488 conjugate (Invitrogen) and further quantified with the FIJI software, using 3 replicates for each genotype. For the AchE analysis, hearts were fixed for 10 minutes in $4 \%$ paraformaldehype and processed for cryosectioning in optimal cutting temperature (OCT) compound. Staining was performed as previously described (62). All images were obtained using Olympus DotSlide.

$M R I$, microCT, and electrocardiogram. Adult mice were anesthetized with isoflurane for noninvasive imaging in a prone position before surgical procedure. Imaging was performed in spontaneously breathing animals on a 9.4 Tesla MRI scanner (Agilent) to assess left and right ventricular chamber dimensions using cardiac and respiration gated cine sequences. Breathing was monitored to avoid measurement distortions during breathing cycle. Measurements of cardiac function were performed using the Segment software (63).

For microCT analysis, 18.5 days after coitum, embryos were dissected and exsanguinated through the severed umbilicus for 10 minutes at $37^{\circ} \mathrm{C}$ in $\mathrm{Ca}^{2+} / \mathrm{Mg}^{2+}$-free PBS containing $0.02 \%$ heparin followed by immersion in $60 \mathrm{mM} \mathrm{KCl}$. Embryos were fixed overnight at $4^{\circ} \mathrm{C}$ in $4 \%$ paraformaldehyde and stored at $4{ }^{\circ} \mathrm{C}$ in $\mathrm{PBS} / 0.02 \%$ sodium azide. To stabilize volume during imaging, embryos were incubated in hydrogel solution (4\% paraformaldehyde [Electron Microscopy Sciences], 4\% acrylamide [Bio-Rad], 0.05\% bis-acrylamide [Bio-Rad], 0.25\% VA044 Initiator [Wako Chemicals], 0.05\% saponin [Sigma-Aldrich] in PBS) at $4{ }^{\circ} \mathrm{C}$ for 3 days. After incubation, air in the tube was replaced with nitrogen gas and the gel polymerized at $37^{\circ} \mathrm{C}$ for 3 hours. Samples were manually separated from the encasing gel and stained in Lugol's solution (Sigma-Aldrich) for 24 hours. The iodine-stained embryo was embedded in 1\% agarose (Lonza) in an 11-mm Ultra-Clear centrifuge tube (Beckman Coulter) for scanning. Datasets were acquired using a Skyscan 1172 high-resolution microCT scanner (Bruker), at $100 \mathrm{kVp}$ and $100 \mu \mathrm{A}, 0.5-\mathrm{mm}$ aluminium filter, step size of $0.3^{\circ}$, averaging of 9 , and pixel size of 13.4 $\mu \mathrm{m}$. Data were reconstructed using Bruker NRecon software with misalignment compensation and ring artifact corrections as needed.

Electrocardiograms (ECG) were obtained on nonanesthetized mice using the ECGenie system (Mouse Specifics) and intervals calculated using EzCG software (Mouse Specifics).For telemetry analysis, 30-weekold male mice from each group were implanted with radiotelemetry probes into the left carotid artery (TA11PA-C10, Data Science International) under anesthesia with isoflurane. Mice were allowed to recover for 1 week, followed by 24-hour monitoring of blood pressure and ECG.

Atrial septal morphological analysis. To detect patent foramen ovale and atrial septal defect, adult mouse hearts were dissected and visually inspected under a stereoscope. Briefly, the left atrial appendage was removed to expose the atrial septum and record blood flow induced by pressurizing the right atrium, as previously described (24). Septa with normal morphology but a patent flap valve were scored as patent foramen ovales. Direct overlap of the ostium secundum and the foramen ovale was scored as an atrial septal defect (24). Excess or hyperelastic tissue over the foramen ovale, capable of protruding significantly when pressurising the right atrium, was scored as atrial aneurysm. 
Cell culture. The mouse atrial cardiomyocyte HL-1 cell line was grown in Claycomb media (Sigma-Aldrich) supplemented with 10\% FBS (Gibco) and norepinephrine (Sigma-Aldrich), maintained in a humidified $5 \% \mathrm{CO}_{2}$ incubator at $37^{\circ} \mathrm{C}$. Cell lines were electroporated (NEON system, Invitrogen) following manufacturer's instructions.

Imaging and photoactivatable flourescence correlation spectroscopy (paFCS) measurements. Nkx2-5paGFP constructs were generated with In-Fusion HD Cloning kit (Clontech) with PCR fragments from Nkx2-5 WT/mutant expression plasmids (22) fused to a photoactivatable form of GFP (paGFP) as previously described for Oct4 (primers described in Supplemental Table 5; ref. 28). HL-1 cells were electroporated as described above using Neon Transfection System (Invitrogen). Cells were cultured in Lab-Tek chambers (Nunc) with $0.1 \%$ gelatin and imaged 72 hours after elecroporation. Zeiss LSM 780 laser scanning confocal microscope/avalanche photodiodes of the Confocor 3 module (Zeiss, Jena) was used and analyzed as previously described (28). Cell nuclei were identified by the expression of H2B-RFP. paFCS measurements of WT Nkx2-5 and I-M, I-P mutants transiently transfected into HL-1 showed two protein movement components, a faster Brownian component (associated with free, unbound NKX2-5) and a slower (in the order of milliseconds) highly anomalous component, representing the bound/target searching component).

Neonatal cardiomyocyte preparation and mitochondrial activity. Neonatal cardiomyocytes (postnatal day 2 ) were prepared as previously described (64). Cells $\left(1 \times 10^{5}\right)$ were added to each well in a fibronectin coated 96-well CM plate (Seahorse). After 24 hours, regular media was replaced with fresh media containing $2 \%$ FBS and Wnt3a $(0.6 \mu \mathrm{g} / \mathrm{ml})$ for 48 hours. Mitochondrial activity (65) was assessed using the Seahorse system (Seahorse Bioscience). Final concentrations used for injection were $1 \mu \mathrm{M}$ Oligomycin, $1.75 \mu \mathrm{M}$ FCCP, and $1 \mu \mathrm{M}$ Antimycin-A.

Transfections and Wnt activity measurements. Neonatal cardiomyocytes were transfected using Viromer Yellow (Lipocalix) 48 hours after isolation using TopFlash Wnt reporter plasmids (66). Recombinant Wnt3a (R\&D Systems) was added 5 hours after transfection. Cells were lysed in 1× RLB (Biotium) 24 hours later, and luciferase activity was measured using Firefly \& Renilla Luciferase Assay Kit (Biotium) using a Fluostar Optima (BMG Labtech) plate reader.

Microarray, bioinformatics analysis, and $q P C R$ validation. Triplicate samples from 15 -week adult male hearts were processed for total RNA extraction using the mirVana kit (Invitrogen) and DNAse digested. Samples were further processed at the Medical Genomics Facility and run on Agilent SurePrint G3 mouse gene expression arrays (single color). Raw single-channel signals provided by the Agilent Feature Extraction image analysis software was used for data analysis and deposited at GEO under the access number GSE85902. $\log _{2}$ transformation and normalization was done in $\mathrm{R}$ scripts using public Bioconductor packages (67). Differential analysis was performed using the Bioconductor Limma package (68), which fits a linear model of gene expression. Differentially expressed genes $(P<$ $0.01)$ were retained for fold-change calculation. For transcriptional analysis in embryos, total RNA was isolated from at least three dissected hearts 9.5-day after coitum using RNAqueous4PCR micro kit (Invitrogen), and DNAse digested. cDNA synthesis was performed using the Superscript VILO kit (Invitrogen) following manufacturer's instructions. qPCR reactions were performed using SYBR green master mix (Roche Diagnostics) and analyzed using the LightCycler480 (Roche Diagnostics). At least two individual experiments in triplicate were performed, using the hypoxanthine phosphoribosyltransferase 1 gene (Hprt1) as a normalizer. Primers/probes are described in Supplemental Table 2. In situ hybridization was performed as described (69). Probes were generated by RT-PCR and cloned into pGEMTeasy (Promega). To obtain antisense transcripts, reactions were performed using T7 RNA Polymerase (Roche Diagnostics). Antibodies are listed in Supplemental Table 7. Venn diagrams were generated using BioVenn (70).

Statistics. All results are reported as the mean \pm SEM. Statistical analysis was performed using unpaired, 2-tailed Student's $t$ test or ANOVA using Graphpad Prism 6 (GraphPad Software Inc.). $P<0.05$ was considered significant unless otherwise stated. $\mathrm{P}$ values are presented in the figure legends.

Study Approval. The investigation conforms to the Guide for the Care and Use of Laboratory Animals published by the US National Institutes of Health (NIH Publication No. 85-23, revised 1996) and with requirements under the ethics application MARP-2011-175 (Monash University) and ACUC16011 (The Jackson Laboratory). 


\section{Author contributions}

$\mathrm{MBF}$ performed experiments, prepared the manuscript, and supervised personnel; JCW, AC, JP, OH, CB, TJW, GK, SS, DW, and ES performed experiments; HTN, QW, and MR analyzed computational data; NP, JMD, SAM, DF, MC, JTP, DK, and RPH supervised personnel and provided scientific support; NAR provided scientific support, revised the manuscript, and supervised personnel; MWC designed experimental procedures, performed experiments, prepared the manuscript, and supervised personnel.

\section{Acknowledgments}

We acknowledge the use of Monash University MicroImaging, Monash Biomedical Imaging, and Monash Gene Targeting Facilities. We would also like to thanks Peter Williams for help with mitochondrial data analysis. The Australian Regenerative Medicine Institute is supported by grants from the State Government of Victoria and the Australian Government. This work was also funded by NHMRC-Australia Fellowship to NAR and NHMRC Project grant 1069710 to MWC and NAR, NHMRC/NHF 1049980 CDF to MR, and ARC Stem Cells Australia to NAR. NHMRC Project grants 025008 and 1074386 and The Estate of the Late RT Hall to DF. HTN is supported by the Richard Pratt Fellowship in Prostate Cancer.

Address correspondence to: Mauro W. Costa, The Jackson Laboratory, 600 Main Street, Bar Harbor, Maine 0460, USA. Phone: 207.288.6149; E-mail: mauro.costa@jax.org.

1. Wessels MW, Willems PJ. Genetic factors in non-syndromic congenital heart malformations. Clin Genet. 2010;78(2):103-123.

2. Fahed AC, Gelb BD, Seidman JG, Seidman CE. Genetics of congenital heart disease: the glass half empty. Circ Res. 2013;112(4):707-720.

3. van der Bom T, Zomer AC, Zwinderman AH, Meijboom FJ, Bouma BJ, Mulder BJ. The changing epidemiology of congenital heart disease. Nat Rev Cardiol. 2011;8(1):50-60.

4. Brickner ME, Hillis LD, Lange RA. Congenital heart disease in adults. First of two parts. N Engl J Med. 2000;342(4):256-263.

5. Benson DW. Genetic origins of pediatric heart disease. Pediatr Cardiol. 2010;31(3):422-429.

6. Elliott D, Kirk EP, Schaft D, Harvey RP. NK-2 Class Homeodomain Proteins: Conserved Regulators of Cardiogenesis. In: Rosenthal N, Harvey RP, eds. Heart Development and Regeneration. Boston: Elsevier, 2010:569-597.

7. Lyons I, et al. Myogenic and morphogenetic defects in the heart tubes of murine embryos lacking the homeo box gene Nkx2-5. Genes Dev. 1995;9(13):1654-1666.

8. Tanaka M, Chen Z, Bartunkova S, Yamasaki N, Izumo S. The cardiac homeobox gene Csx/Nkx2.5 lies genetically upstream of multiple genes essential for heart development. Development. 1999;126(6):1269-1280.

9. Briggs LE, et al. Perinatal loss of Nkx2-5 results in rapid conduction and contraction defects. Circ Res. 2008;103(6):580-590.

10. Pashmforoush M, et al. Nkx2-5 pathways and congenital heart disease; loss of ventricular myocyte lineage specification leads to progressive cardiomyopathy and complete heart block. Cell. 2004;117(3):373-386.

11. Takeda M, et al. Slow progressive conduction and contraction defects in loss of Nkx2-5 mice after cardiomyocyte terminal differentiation. Lab Invest. 2009;89(9):983-993.

12. Terada R, Warren S, Lu JT, Chien KR, Wessels A, Kasahara H. Ablation of Nkx2-5 at mid-embryonic stage results in premature lethality and cardiac malformation. Cardiovasc Res. 2011;91(2):289-299.

13. Kasahara $\mathrm{H}$, et al. Progressive atrioventricular conduction defects and heart failure in mice expressing a mutant Csx/Nkx2.5 homeoprotein. J Clin Invest. 2001;108(2):189-201.

14. Schott JJ, et al. Congenital heart disease caused by mutations in the transcription factor NKX2-5. Science. 1998;281(5373):108-111.

15. Benson DW, et al. Mutations in the cardiac transcription factor NKX2.5 affect diverse cardiac developmental pathways. J Clin Invest. 1999;104(11):1567-1573.

16. Elliott DA, et al. Cardiac homeobox gene NKX2-5 mutations and congenital heart disease: associations with atrial septal defect and hypoplastic left heart syndrome. J Am Coll Cardiol. 2003;41(11):2072-2076.

17. Gutierrez-Roelens I, Sluysmans T, Gewillig M, Devriendt K, Vikkula M. Progressive AV-block and anomalous venous return among cardiac anomalies associated with two novel missense mutations in the CSX/NKX2-5 gene. Hum Mutat. 2002;20(1):75-76.

18. König K, Will JC, Berger F, Müller D, Benson DW. Familial congenital heart disease, progressive atrioventricular block and the cardiac homeobox transcription factor gene NKX2.5: identification of a novel mutation. Clin Res Cardiol. 2006;95(9):499-503.

19. Perera JL, Johnson NM, Judge DP, Crosson JE. Novel and highly lethal NKX2.5 missense mutation in a family with sudden death and ventricular arrhythmia. Pediatr Cardiol. 2014;35(7):1206-1212.

20. Goldmuntz E, Geiger E, Benson DW. NKX2.5 mutations in patients with tetralogy of fallot. Circulation. 2001;104(21):2565-2568.

21. Maury P, et al. Cardiac Phenotype and Long-Term Follow-Up of Patients With Mutations in NKX2-5 Gene. J Am Coll Cardiol. 2016;68(21):2389-2390.

22. Costa MW, et al. Functional characterization of a novel mutation in NKX2-5 associated with congenital heart disease and adult-onset cardiomyopathy. Circ Cardiovasc Genet. 2013;6(3):238-247.

23. Hanley A, et al. Mutation of a common amino acid in NKX2.5 results in dilated cardiomyopathy in two large families. $B M C$ Med Genet. 2016;17(1):83.

24. Biben C, et al. Cardiac septal and valvular dysmorphogenesis in mice heterozygous for mutations in the homeobox gene Nkx25. Circ Res. 2000;87(10):888-895. 
25. Kasahara H, Benson DW. Biochemical analyses of eight NKX2.5 homeodomain missense mutations causing atrioventricular block and cardiac anomalies. Cardiovasc Res. 2004;64(1):40-51.

26. Kasahara $\mathrm{H}$, et al. Loss of function and inhibitory effects of human CSX/NKX2.5 homeoprotein mutations associated with congenital heart disease. J Clin Invest. 2000;106(2):299-308.

27. Kasahara H, Usheva A, Ueyama T, Aoki H, Horikoshi N, Izumo S. Characterization of homo- and heterodimerization of cardiac Csx/Nkx2.5 homeoprotein. J Biol Chem. 2001;276(7):4570-4580

28. Kaur G, et al. Probing transcription factor diffusion dynamics in the living mammalian embryo with photoactivatable fluorescence correlation spectroscopy. Nat Commun. 2013;4:1637.

29. Furtado $\mathrm{MB}$, et al. A novel conditional mouse model for $\mathrm{Nkx} 2-5$ reveals transcriptional regulation of cardiac ion channels. Differentiation. 2016;91(1-3):29-41.

30. Reamon-Buettner SM, Borlak J. NKX2-5: an update on this hypermutable homeodomain protein and its role in human congenital heart disease (CHD). Hum Mutat. 2010;31(11):1185-1194.

31. Mommersteeg MT, et al. Pitx2c and Nkx2-5 are required for the formation and identity of the pulmonary myocardium. Circ Res 2007;101(9):902-909.

32. Prall OW, et al. An Nkx2-5/Bmp2/Smad1 negative feedback loop controls heart progenitor specification and proliferation. Cell. 2007;128(5):947-959.

33. Hirayama-Yamada K, et al. Phenotypes with GATA4 or NKX2.5 mutations in familial atrial septal defect. Am J Med Genet A. 2005;135(1):47-52.

34. Sarkozy A, et al. Spectrum of atrial septal defects associated with mutations of NKX2.5 and GATA4 transcription factors. J Med Genet. 2005;42(2):e16.

35. Bouveret R, et al. NKX2-5 mutations causative for congenital heart disease retain functionality and are directed to hundreds of targets. Elife. 2015;4.

36. Calvo SE, Clauser KR, Mootha VK. MitoCarta2.0: an updated inventory of mammalian mitochondrial proteins. Nucleic Acids Res. 2016;44(D1):D1251-D1257.

37. Hermans KC, Blankesteijn WM. Wnt Signaling in Cardiac Disease. Compr Physiol. 2015;5(3):1183-1209.

38. Jain R, et al. HEART DEVELOPMENT. Integration of Bmp and Wnt signaling by Hopx specifies commitment of cardiomyoblasts. Science. 2015;348(6242):aaa6071.

39. Blow MJ, et al. ChIP-Seq identification of weakly conserved heart enhancers. Nat Genet. 2010;42(9):806-810.

40. He A, Kong SW, Ma Q, Pu WT. Co-occupancy by multiple cardiac transcription factors identifies transcriptional enhancers active in heart. Proc Natl Acad Sci USA. 2011;108(14):5632-5637.

41. Ravasi T, et al. An atlas of combinatorial transcriptional regulation in mouse and man. Cell. 2010;140(5):744-752

42. Schlesinger J, et al. The cardiac transcription network modulated by Gata4, Mef2a, Nkx2.5, Srf, histone modifications, and microRNAs. PLoS Genet. 2011;7(2):e1001313.

43. Bruneau BG, et al. A murine model of Holt-Oram syndrome defines roles of the T-box transcription factor Tbx 5 in cardiogenesis and disease. Cell. 2001;106(6):709-721.

44. Ashraf $\mathrm{H}$, et al. A mouse model of human congenital heart disease: high incidence of diverse cardiac anomalies and ventricular noncompaction produced by heterozygous Nkx2-5 homeodomain missense mutation. Circ Cardiovasc Genet. 2014;7(4):423-433.

45. Abou Hassan OK, et al. NKX2-5 mutations in an inbred consanguineous population: genetic and phenotypic diversity. Sci Rep. 2015;5:8848.

46. Winston JB, et al. Heterogeneity of genetic modifiers ensures normal cardiac development. Circulation. 2010;121(11):1313-1321

47. Winston JB, et al. Complex trait analysis of ventricular septal defects caused by Nkx2-5 mutation. Circ Cardiovasc Genet. 2012;5(3):293-300.

48. Davlouros PA, Niwa K, Webb G, Gatzoulis MA. The right ventricle in congenital heart disease. Heart. 2006;92 Suppl 1:i27-i38

49. Paffett-Lugassy N, et al. Heart field origin of great vessel precursors relies on nkx2.5-mediated vasculogenesis. Nat Cell Biol. 2013;15(11):1362-1369.

50. Chowdhury R, et al. Mouse Model of Human Congenital Heart Disease: Progressive Atrioventricular Block Induced by a Heterozygous Nkx2-5 Homeodomain Missense Mutation. Circ Arrhythm Electrophysiol. 2015;8(5):1255-1264.

51. Ozhan G, Weidinger G. Wnt/ $\beta$-catenin signaling in heart regeneration. Cell Regen (Lond). 2015;4(1):3

52. Liang W, Cho HC, Marbán E. Wnt signalling suppresses voltage-dependent $\mathrm{Na}+$ channel expression in postnatal rat cardiomyocytes. J Physiol (Lond). 2015;593(5):1147-1157.

53. Baurand A, et al. Beta-catenin downregulation is required for adaptive cardiac remodeling. Circ Res. 2007;100(9):1353-1362.

54. Deb A. Cell-cell interaction in the heart via Wnt/ $\beta$-catenin pathway after cardiac injury. Cardiovasc Res. 2014;102(2):214-223.

55 . Duan J, et al. Wnt1/ $\beta$ catenin injury response activates the epicardium and cardiac fibroblasts to promote cardiac repair. $E M B O$ J. 2012;31(2):429-442.

56. Riazi AM, et al. NKX2-5 regulates the expression of beta-catenin and GATA4 in ventricular myocytes. PLoS One. 2009;4(5):e5698.

57. Ouyang P, et al. A de novo mutation in NKX2.5 associated with atrial septal defects, ventricular noncompaction, syncope and sudden death. Clin Chim Acta. 2011;412(1-2):170-175.

58. Scaglia F, et al. Clinical spectrum, morbidity, and mortality in 113 pediatric patients with mitochondrial disease. Pediatrics 2004;114(4):925-931.

59. Towbin JA, Lorts A, Jefferies JL. Left ventricular non-compaction cardiomyopathy. Lancet. 2015;386(9995):813-825.

60. Yuan S, Zaidi S, Brueckner M. Congenital heart disease: emerging themes linking genetics and development. Curr Opin Genet Dev. 2013;23(3):352-359.

61. Schwenk F, Baron U, Rajewsky K. A cre-transgenic mouse strain for the ubiquitous deletion of loxP-flanked gene segments including deletion in germ cells. Nucleic Acids Res. 1995;23(24):5080-5081.

62. El-Badawi A, Schenk EA. Histochemical methods for separate, consecutive and simultaneous demonstration of acetylcholinesterase and norepinephrine in cryostat sections. J Histochem Cytochem. 1967;15(10):580-588.

63. Heiberg E, Sjögren J, Ugander M, Carlsson M, Engblom H, Arheden H. Design and validation of Segment--freely available 
software for cardiovascular image analysis. BMC Med Imaging. 2010;10:1.

64. Durocher D, Charron F, Warren R, Schwartz RJ, Nemer M. The cardiac transcription factors Nkx2-5 and GATA-4 are mutual cofactors. EMBO J. 1997;16(18):5687-5696.

65. Brand MD, Nicholls DG. Assessing mitochondrial dysfunction in cells. Biochem J. 2011;435(2):297-312.

66. Veeman MT, Slusarski DC, Kaykas A, Louie SH, Moon RT. Zebrafish prickle, a modulator of noncanonical Wnt/Fz signaling, regulates gastrulation movements. Curr Biol. 2003;13(8):680-685.

67. Gentleman RC, et al. Bioconductor: open software development for computational biology and bioinformatics. Genome Biol. 2004;5(10):R80

68. Smyth GK, Michaud J, Scott HS. Use of within-array replicate spots for assessing differential expression in microarray experiments. Bioinformatics. 2005;21(9):2067-2075.

69. Biben C, Harvey RP. Homeodomain factor Nkx2-5 controls left/right asymmetric expression of bHLH gene eHand during murine heart development. Genes Dev. 1997;11(11):1357-1369.

70. Hulsen T, de Vlieg J, Alkema W. BioVenn - a web application for the comparison and visualization of biological lists using area-proportional Venn diagrams. BMC Genomics. 2008;9:488 